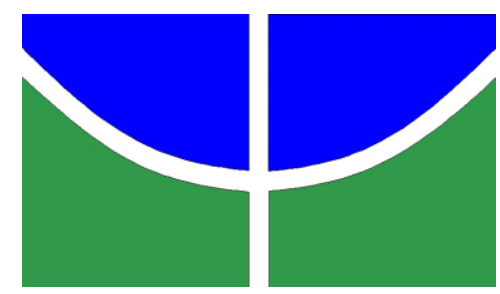

Universidade de Brasília

Faculdade de Educação Física

Programa de Pós-Graduação em Educação Física

Mauricio Andres Ardiles Eguíluz

Efeitos da Equoterapia e Fisioterapia Convencional na força muscular de idosos com doença de Alzheimer.

Brasília, Dezembro de 2016. 
Mauricio Andres Ardiles Eguíluz

Efeitos da Equoterapia e Fisioterapia Convencional na força muscular de idosos com doença de Alzheimer.

Exame de Defesa de Dissertação de Mestrado, apresentado à banca examinadora, como requisito de avaliação para obtenção do título de Mestre em Educação Física pela Universidade de Brasília.

Orientadora: Profa. Dra. Marisete Peralta Safons

Brasília, Dezembro de 2016. 
Mauricio Andres Ardiles Eguíluz

\section{Efeitos da Equoterapia e Fisioterapia Convencional na força muscular de idosos com doença de Alzheimer.}

Exame de Defesa de Dissertação de Mestrado, apresentado à banca examinadora, como requisito de avaliação para obtenção do título de Mestre em Educação Física pela Universidade de Brasília.

Banca Examinadora

Profa. Profa. Dra. Marisete Peralta Safons

(Orientadora-FEF/UnB)

Prof. Dr. Paulo Gutierres Filho

(Examinador interno-FEF/UnB)

Prof. Dr. Alexandre Rezende

(Examinador externo-FEF/UnB)

Data de aprovação: 12/12/2016

Brasília, Dezembro de 2016. 


\section{Dedicatória}

Dedico este trabalho à minha família do Chile e a do Brasil, pelo incentivo, amor e carinho. 


\section{Agradecimentos}

A Deus, pelo cuidado, acompanhamento e força em todo momento. Aos meus pais, Mauricio Javier Ardiles Rios e Gina Fiorella Eguíluz Castillo, através dos quais recebi sempre o amor e apoio que precisei, e a minha irmã Loreto Ardiles Eguíluz, por ser fonte de inspiração para os estudos.

A minha querida esposa, Vicki Araújo Passos Ardiles, amiga e cúmplice, agradeço o seu amor, carinho, apoio, sua motivação e por estar presente em todos os instantes. Por me ajudar e por fazer possível minha vinda para o Brasil, sem sua gestão não teria conseguido realizar minhas metas. Simplesmente amo você!

A minha filha Carolina Araújo Ardiles e aos meus enteados Eduardo e Maria Luiza, por me tornarem uma pessoa cada dia melhor, a minha cunhada Isabella Araujo Goellner pela força nos últimos momentos.

A minha orientadora, à professora Dra. Marisete Safons, que me ofereceu essa oportunidade de aprendizado e amadurecimento; por todo seu apoio e incentivo, cobrando sempre no momento certo. Á doutoranda Thais Borges de Araújo por me permitir fazer parte deste projeto, esse trabalho é fruto de nosso esforço e sacrifício, agradeço por contribuir tanto com o meu crescimento profissional quanto pessoal.

Ao pessoal do Centro de Medicina do Idoso, pelo auxílio no recrutamento dos idosos, ao pessoal da ANDE Brasil pelo apoio logístico durante a intervenção da pesquisa.

A todos os professores com os quais já tive o prazer de aprender, aos professores responsáveis pelos laboratórios, aos funcionários da Faculdade de Educação Física e do Centro Olímpico, por estarem sempre dispostos a ajudar, sem esperar nada em troca.

Às agências financiadoras CAPES e CNPq.

Enfim, a todos que colaboraram, ofereço o meu sincero:

Obrigado! 


\section{Resumo}

O uso de diferentes Programas de Tratamento como são a Equoterapia e a Fisioterapia Convencional são consideradas alternativas viáveis no ganho de força muscular nos membros inferiores na população idosa. No entanto, ainda são escassos os trabalhos que investigam os efeitos da Equoterapia e Fisioterapia na população idosa com demência de Alzheimer. A proposta deste estudo foi avaliar 12 semanas de um programa de Equoterapia e um programa de Fisioterapia (duas vezes por semana) e os efeitos que produzem na força muscular dos membros inferiores em mulheres e homens idosos com demência de Alzheimer. Dezessete (17) homens e mulheres idosos foram alocados em dois grupos, Grupo Equoterapia (GE) e Grupo Fisioterapia (GF). Dezessete participantes (17) no total finalizaram a pesquisa. No GE, com médias de idade de $74.4 \pm 3.3$ anos, Estado Cognitivo (Minimental) $21.1 \pm 2.6$ pontos, Indice de Barthel de $93.5 \pm 11.8$ pontos. No GF, com médias de idade de $76.1 \pm 2.3$ anos, Estado Cognitivo (Minimental) $21.7 \pm 2.2$ pontos, Indice de Barthel de $92.1 \pm 8.6$ pontos. Foram as variáveis mensuradas nos momentos PRÉ é PÓS; o pico de torque para extensores e flexores de joelho (PT) na velocidade de $60 \%$, respetivamente. Referente à força muscular, os membros inferiores na velocidade de $60 \% \mathrm{~s}$ e em ambos grupos obtiveram ganhos, sendo estatisticamente significativo $(p<0.05)$, a força de flexão do joelho para ambos gupos $(p=0.0001)$. Os dois grupos não apresentaram diferenças estatísticas, no que refere ao pico de torque na extensão de joelho ( $p=0.893$ ) no momento pós intervenção. Em adição, o uso da Equoterapia e Fisioterapia pode constituir uma importante ferramenta de intervenção para atenuar os efeitos deletérios associados ao envelhecimento em idosos com demência de Alzheimer.

Palavras-chave: Equoterapia. Força muscular. Alzheimer. Fisioterapia. Dinamômetro isocinético 


\begin{abstract}
The use of different Treatment Programs such as Hippotherapy and Conventional Physical Therapy are considered potential alternatives in the gain of muscle strength in the lower limbs in the elderly population. However, there are still few studies that investigate the effects of Hippotherapy and Conventional Physical Therapy in the elderly population with Alzheimer's dementia. The purpose of this study was to evaluate 12 weeks of an Hippotherapy program and Conventional Physical Therapy program (twice a week) and the effects they produce on lower limb muscle strength in elderly women and men with Alzheimer's dementia. Seventeen (17) elderly men and women were allocated to two groups, Group Equoterapia (GE) and Group Physiotherapy (GF). Seventeen participants (17) in total completed the survey. In the GE, with a mean age of $74.4 \pm 3.3$ years, Cognitive Status (Minimental) $21.1 \pm 2.6$ points, Barthel Index of $93.5 \pm 11.8$ points. In GF, with a mean age of $76.1 \pm 2.3$ years, Cognitive Status (Minimental) $21.7 \pm 2.2$ points, Barthel Index of $92.1 \pm 8.6$ points. The variables measured in the moments PRÉ é PÓS; Peak torque for knee extensors and flexors (PT) at the rate of $60 \%$ s, respectively. Regarding muscle strength, the knee flexion strength for both groups $(\mathrm{p}=0.0001)$ was statistically significant $(\mathrm{p}<0.05)$ at the $60 \%$ s velocity and in both groups. The two groups did not present statistical differences, regarding the peak torque in the knee extension $(p=0.893)$ at the moment after intervention. In addition, the use of Hippotherapy and Conventional Physical Therapy can be an important intervention tool to attenuate the deleterious effects associated with aging in the elderly with Alzheimer's dementia.
\end{abstract}

Keywords: Hippotherapy. Muscle strength. Alzheimer. Physical Therapy. Isokinetic Dynamometer 


\section{Sumário}

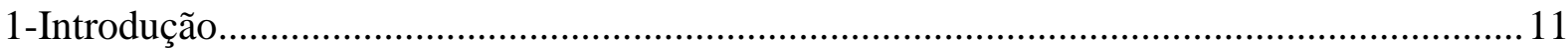

2. Objetivos

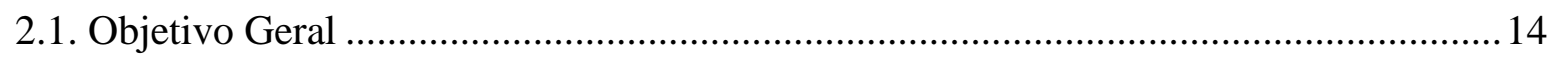

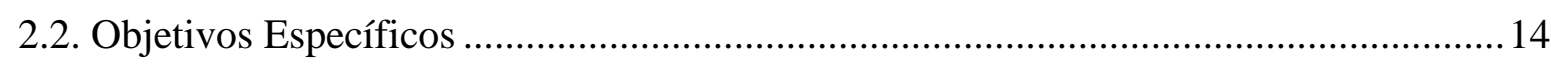

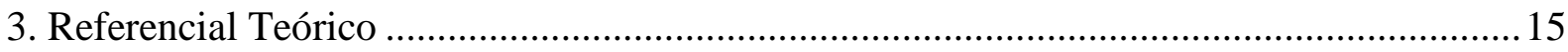

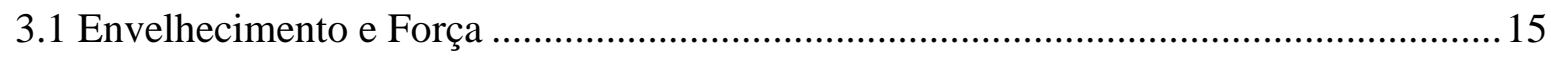

3.2.Doença de Alzheimer e a deterioração físico/funcional ................................................... 18

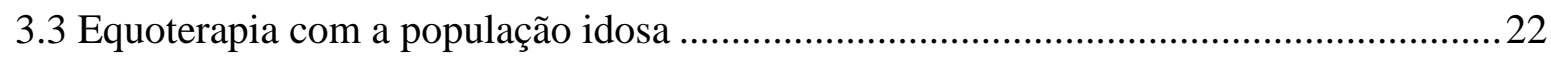

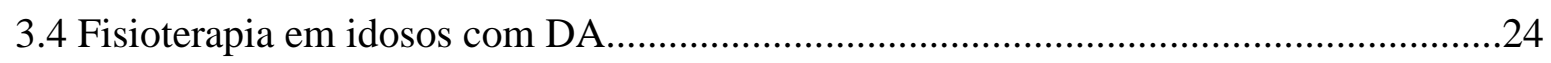

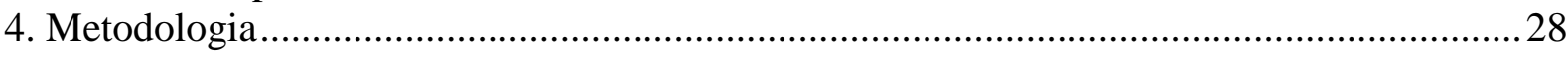

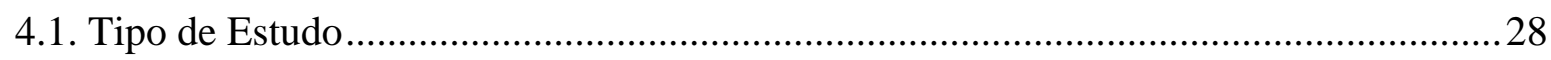

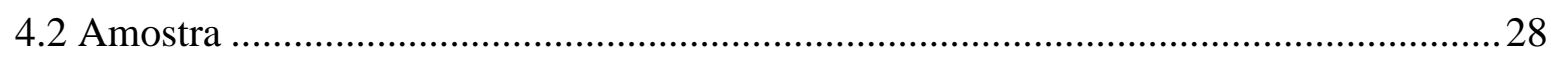

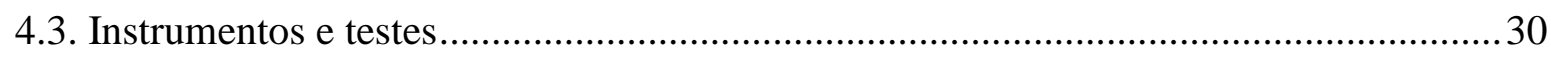

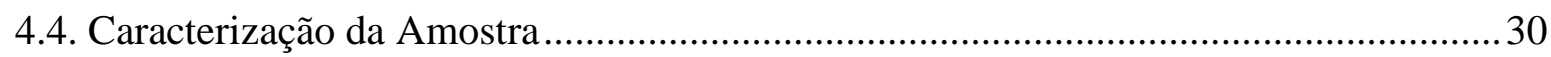

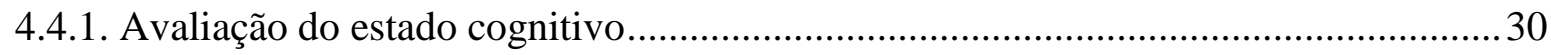

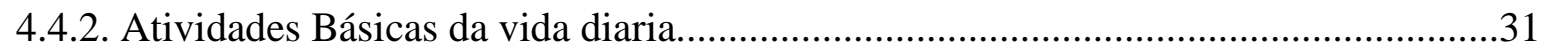

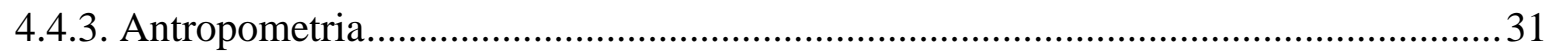

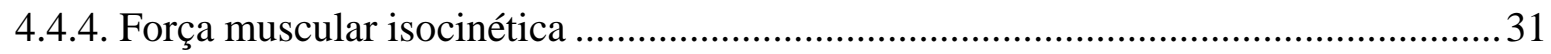

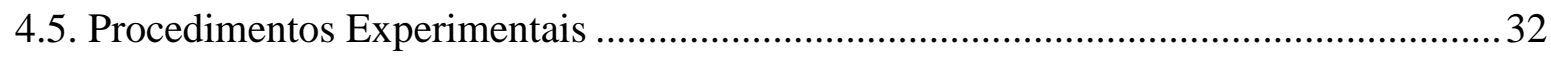

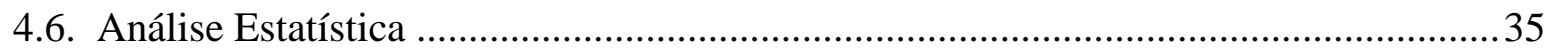

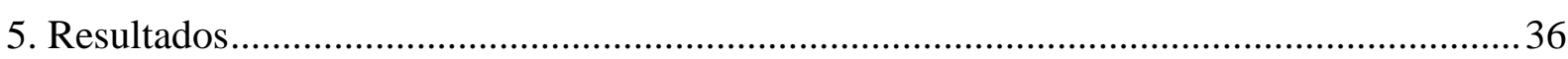

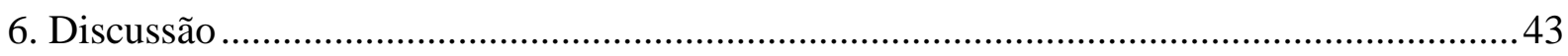

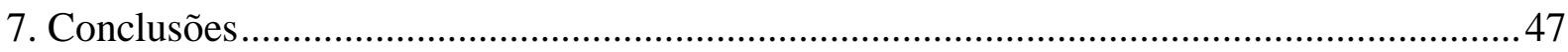

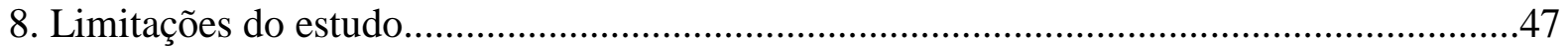

9. Referências Bibliográficas.............................................................................................. 


\section{Lista de ilustrações}

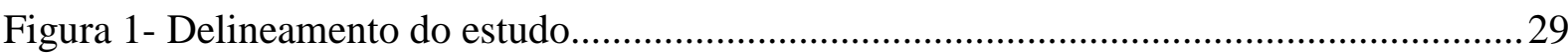

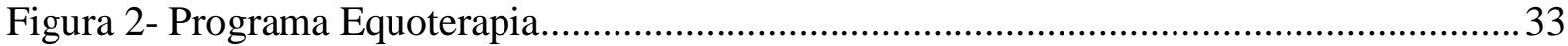

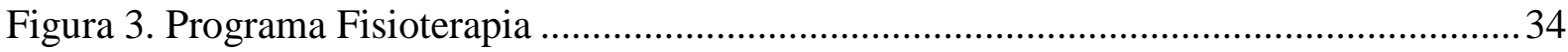




\section{Lista de tabelas}

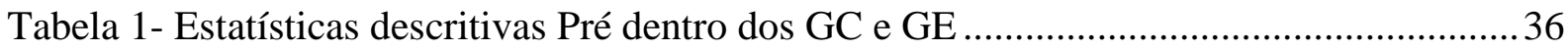

Tabela 2- Média \pm Desvio Padrão - Pré X Pós Dentro Dos Grupos ........................................... 37

Tabela 3-Teste Anova (Split-plot) para amostras pareadas - Pré x Pós dentro dos grupos...... 38

Tabela 4-Teste Anova (Split-plot) para amostras pareadas - Pré x Pós dentro dos grupos...... 40

Tabela 5-Teste Mcnemar para o efeito na dependência funcional...........................................42 


\section{Lista de Gráficos}

Gráfico 1-Estimação de Médias Marginais para flexão de joelho............................................39

Gráfico 2-Estimação de Médias Marginais para extensão de joelho........................................ 41 


\section{Lista de abreviações}

IBGE- Instituo Brasileiro de Geografia e Estatística

DA- Doença de Alzheimer

ABVD- Atividades Básicas da Vida Diária

PE- Programa de Equoterapia

PF- Programa de Fisioterapia

HUB- Hospital Universitário de Brasília

CMI- Centro de Medicina do Idoso

DF- Distrito Federal

IMC- Índice de massa corporal

MMSE- Minimental Teste

PRÉ- Pré-intervenção

PÓS- Pós-intervenção

GE- Grupo Equoterapia

GF- Grupo Fisioterapia

PT- Pico de Torque 


\section{1-Introdução}

O envelhecimento populacional é um fenômeno global. Conceitualmente, ele pode ser definido como uma mudança na estrutura etária da população que resulta em uma maior proporção de idosos (CARVALHO e GARCIA, 2003). No Brasil, esse processo teve início na década de 60 devido a uma significativa redução da taxa de fecundidade e ao aumento da longevidade, o qual está relacionado aos avanços na medicina, à melhoria da saúde pública, às melhores condições de alimentação, ao aumento da renda e ao controle de vetores causadores de doenças infecciosas (BLOOM, 2011). De acordo com dados do Instituto Brasileiro de Geografia e Estatística (IBGE), a estimativa é que até o ano de 2060, o número de idosos chegue a 73 milhões (IBGE, 2012).

Com o aumento da população idosa, ocorre um incremento na prevalência de doenças crônicas não transmissíveis, as quais se prolongam por vários anos e exigem acompanhamento constante (QUEIROZ, LEMOS e RAMOS, 2010). Dentre as doenças crônicas que acometem essa população, tem-se a Doença de Alzheimer (DA), uma doença de caráter neurodegenerativo e progressivo, caracterizada por alterações comportamentais e cognitivas, incluindo o comprometimento da memória, do pensamento e do raciocínio (REITZ, BRAYNE e MAYEUX, 2011).

Atualmente, a DA acomete uma parcela considerável dos idosos em diversos países do mundo. Nos Estados Unidos, $3 \%$ a $11 \%$ das pessoas com idade superior a 65 anos, e $25 \%$ a 47\% daquelas com mais de 85 anos apresentam demência (REITZ, BRAYNE e MAYEUX, 2011). Em nosso país, a estatística é semelhante à mundial. Estudo de base populacional conduzido em Catanduva, município com 100 mil habitantes do estado de São Paulo, verificou a presença de demência em $25 \%$ dos idosos avaliados, sendo que em aproximadamente metade dos casos o diagnóstico foi de DA (APRAHAMIAN, MARTINELLI e YASSUDA, 2009).

Além da elevada prevalência, a DA é especialmente preocupante por acarretar em perda gradativa da autonomia, levando a uma situação de dependência total em que há múltiplas demandas e custos financeiros elevados (ABREU, BARROS e FORLENZA, 2005). Com a progressão da DA, o indivíduo adquire importantes comprometimentos motores, como perda da informação proprioceptiva, lentidão no processamento cognitivo e dificuldade na execução da resposta motora exigida (MAKI e MCILROY, 1996; DUNCAN et al., 1993). Há ainda alterações inerentes ao próprio processo de envelhecimento, como perda progressiva da massa muscular esquelética (sarcopenia) e aumento no tecido adiposo intramuscular (TROMBETTI 
et al., 2016). Dessa forma, o idoso com DA exibe uma diminuição na força muscular e consequente limitação funcional.

Para minimizar o declínio funcional, o tratamento fisioterapêutico é indicado. Este tratamento é baseado no quadro-clínico do paciente e costuma ser recomendado para prevenir encurtamentos da musculatura e dos tendões, evitar deformidades articulares, manter o equilíbrio, melhorar o padrão de marcha e estimular a independência do idoso (DE MELO e DRIUSSO, 2006). Contudo, ainda são escassas as evidências científicas acerca da eficácia da fisioterapia convencional no tratamento dos agravos motores relacionados à DA (DE AQUINO et al., 2013). Nesse contexto, foi encontrado apenas o estudo de Ahn e Kim (2015), o qual observou que um programa de treinamento com resistência elástica (3x/sem, 5 meses) foi capaz de promover melhora significativa na função muscular de indivíduos com DA.

Outro método terapêutico que pode ser promissor para o tratamento da DA é a equoterapia - uma técnica que utiliza o cavalo como um agente promotor de ganhos físicos e psicológicos ao paciente (CIRILLO, 2002). A nível físico, o cavalo possui ciclos de movimentação análogos aos ciclos da marcha humana. Quando o cavalo se desloca, é exigida do praticante a adaptação da sua postura a cada movimento; e por trabalhar todo o corpo, colabora para o desenvolvimento da força e tônus muscular, flexibilidade, relaxamento, consciência corporal e aperfeiçoamento da coordenação motora e do equilíbrio (BENDA, MCGIBBON e GRANT, 2003; COPETTI et al., 2007). A nível psicológico, o praticante e o cavalo criam um relacionamento afetivo importante, onde se estabelece uma relação harmoniosa, que atua como um agente motivacional para o engajamento no tratamento (CIRILLO, 2002). Essas características, em conjunto, podem ser benéficas para a função muscular de indivíduo com DA.

Em extensa revisão de literatura, no entanto, não foram encontrados estudos que avaliassem os efeitos da equoterapia em idosos com DA. Estudo conduzido em idosos saudáveis, porém, apontam para resultados promissores. Araujo et al. (2013), por exemplo, conduziram um programa de Equoterapia com duração de oito semanas, observando um aumento significativo da força muscular de membros inferiores de idosos saudáveis, avaliados pelo teste de sentar e levantar da cadeira em 30 segundos.

Diante dos benefícios da equoterapia em outras populações, e da relação da força muscular com a manutenção da independência funcional, surgiu o interesse para delinear uma intervenção com equoterapia em idosos com DA e verificar suas implicações sobre a força muscular dessa população. Sendo assim, o objetivo do presente estudo foi verificar se dois 
programas de exercícios terapêuticos (Equoterapia e Fisioterapia Convencional) melhoram a força muscular de membros inferiores em idosos com DA na fase leve e moderada. 


\section{Objetivos}

\subsection{Objetivo Geral}

Verificar se dois programas de exercícios terapêuticos distintos com são a Equoterapia e a Fisioterapia Convencional melhoram a força muscular de membros inferiores de idosos com DA fase leve e moderada.

\subsection{Objetivos Específicos}

I. Analisar os efeito de um Programa de Equoterapia de 12 semanas no pico de torque de extensão e flexão do joelho em idosos com demência de Alzheimer.

II. Analisar os efeitos de um Programa de Fisioterapia Convencional de 12 semanas no pico de torque de extensão e flexão do joelho em idosos com demência de Alzheimer. 


\section{Referencial Teórico}

\subsection{Envelhecimento e Força}

A associação entre o envelhecimento e a diminuição da força muscular vem sendo estudada a mais de 150 anos. Atualmente, existe o consenso que a diminuição da força se torna mais evidente a partir dos 60 anos, além, de ser mais acentuada no sexo feminino pós-menopausa (PHILLIPS, S. K. et al. 1993, VUKSANOVIC, M. et al. 2016). De acordo com estudo promovido por Deschenes, a força muscular máxima é alcançada por volta dos 30 anos de idade, mantendo-se mais ou menos estável até à quinta década, idade, a partir da qual, inicia-se o seu declínio. Entre os 50 e os 70 anos de idade, existe uma perda de aproximadamente $15 \%$ da força muscular por década vivida, após essa idade, a diminuição passa a ser de $30 \%$ a cada dez anos (CARVALHO J., SOARES J., DESCHENES 2004).

O envelhecimento biológico está associado ao declínio da função e da morfologia neuromuscular, resultando na diminuição da força máxima, potência e do tempo de reação neuromuscular. Os fatores que diminuem a força muscular são as mudanças no padrão neural de recrutamento de unidades motoras, os declínios na taxa de aceleração máxima e na sincronização de unidades motoras. As alterações morfológicas estão associadas principalmente com à atrofia da fibra muscular, com as consequentes reduções na área de secção transversa e espessura muscular, especialmente em fibras Tipo II, que têm a maior unidade motora de força, potência e velocidade de contração (CORREA et al, 2012).

A diminuição da força não é apenas específica em cada indivíduo, mas também em cada grupo muscular e, ainda, em cada tipo de contração muscular. Encontra-se, na literatura, a descrição das alterações nas propriedades contrácteis (tempo para alcançar pico máximo TPM; semi-tempo de relaxamento - 1/2 TR; velocidade máxima de encurtamento - Vmax torque máximo) de diferentes grupos musculares. Por exemplo, em estudo realizado por Grimby, mostrou-se que a diminuição da força dos membros inferiores é mais acentuada do que a observada nos membros superiores quando envelhecemos (GRIMBY G. et al., 1992). Assim outra possibilidade implicada no processo de diminuição da força muscular é o fato dos idosos terem uma reduzida capacidade de ativar completamente os seus grupos musculares (YUE G., et al., 1999).

O declínio muscular é provocado por uma contração mais duradoura, um relaxamento lento e aumento da fatigabilidade muscular, bem como os fatores neurais, que levam à redução da excitabilidade, diminuição do recrutamento motor, e mudanças na estrutura da actina e miosina (BRIAN CLARK; TODD MANINI, 2011). 
O declínio na estrutura do sistema muscular tem implicações significativas na capacidade funcional do idoso. Tem-se demonstrado que existe uma correlação positiva da força muscular - particularmente a força dos extensores do joelho -, com a velocidade da marcha, com a subida de degraus, com a capacidade de se levantar de uma cadeira e com a capacidade de realizar diferentes atividades da vida diária (HYATT R., et al., 1990).

No estudo de Avlund et al. 1994, idosos com idade média de 75 anos, que apresentavam reduzidos níveis de força nos músculos extensores do joelho, mostraram, também, uma maior fatigabilidade, bem como uma maior necessidade de ajuda na realização de diferentes atividades da vida diária.

Aniansson et al. 1992, descreve em seus estudos, que acompanharam por um período de onze anos, nove indivíduos do sexo masculino com idade entre 79 e 82 anos de idade, e constataram uma redução de $35 \%$ da força para contração isométrica e $25 \%$ para contração isocinética para extensores dos joelhos.

Assim, a diminuição da força muscular, particularmente dos membros inferiores, relacionase não apenas com o declínio do equilíbrio mas, igualmente, com a qualidade da marcha (KWON et al., 2001).

A incapacidade de andar regularmente, reduz a aptidão física e impõe um estilo de vida sedentário. Destarte, um estilo de vida excessivamente sedentário leva a graves alterações fisiopatológicas, como a atrofia muscular, o comprometimento do equilíbrio, a hipotensão ortostática e o comprometimento da função cardiorrespiratória, trazendo, como consequências psicológicas: apatia, depressão e declínio cognitivo (BLOCKER J.R., 1992).

Portanto, pelo exposto, a fraqueza muscular contribui para alterações na mobilidade e na autonomia, gerando maior risco de quedas e fraturas. Contudo, um adequado programa de treino de força pode constituir-se como meio importante para a vida diária do idoso, vez que níveis moderados de força são necessários para a realização de inúmeras tarefas diárias, tais como: carregar pesos, subir escadas, levantar-se de cadeiras, etc. Neste sentido, a força adquire importância cada vez mais acentuada com o avançar da idade.

O treinamento da força muscular do membro inferior, por exemplo, no quadríceps femoral, gastrocnêmio e bíceps femoral, tem o potencial de melhorar a função motora, propiciando melhor equilíbrio e mobilidade (LAROCHE, D. P.; MILLETT, E. D.; KRALIAN, R. J, 2011).

Bossers realizou pesquisa comparativa entre três grupos de idosos, sendo: um grupo de controle que não realizou exercícios; um grupo que realizou treinamento aeróbico e de força; e, um grupo que realizou apenas treinamento aeróbico. Como resultado, obteve-se a conclusão de 
que é mais eficaz a combinação de treinos do que apenas o treinamento aeróbico por si só, para retardar o declínio cognitivo e motor em doentes com demência (BOSSERS W.J et al, 2014).

O músculo esquelético de idosos pode sofrer adaptações morfológicas e fisiológicas importantes com a prática do treinamento da força, que pode ser caracterizada pelo maior recrutamento das unidades motoras, aumento da área de secção transversa do músculo e adaptações metabólicas (HUNTER, G. R.; MCCARTHY, J. P.; BAMMAN, M. M, 2004), aumento da capilarização da musculatura esquelética, aumento dos níveis das enzimas oxidativas da musculatura, redução da resistência muscular esquelética e aumento do diâmetro dos vasos sanguíneos (SPIRDUSO, 2005).

MacDonald pondera que, embora não haja consenso de que a disfunção neuronal motora pode levar a diminuições na massa muscular (Sarcopenia) e força, uma associação inversa - via declínio da participação em atividade física ou processo comum subjacente - não pode ser descartada (MACDONALD, S. WS; DECARLO, C. A.; DIXON, R., 2011).

Peterson et al. 2010, realizou metanálise, comparando 47 estudos, com 78 programas de treinamento diversos, totalizando 1.079 idosos saudáveis de ambos os sexos. Nessa pesquisa, foram observadas mudanças significativas, confrontando pré e pós-intervenção, na força dos membros inferiores e superiores, através de diferentes grupos musculares avaliados pelo teste de repetição máxima (1RM). Os resultados sugerem que o treinamento resistido é eficaz para melhorar a força entre adultos mais velhos, particularmente o treinamento com maior intensidade, sendo considerado estratégia viável para evitar a fraqueza muscular generalizada, associada ao envelhecimento.

A compreensão desta realidade pode ajudar na construção de estratégias de intervenção para a população idosa, visando diminuir a perda de força, melhorando assim, a mobilidade e autonomia. 


\subsection{Doença de Alzheimer e a deterioração físico/funcional}

A demência é uma condição crônica, caracterizada por um comprometimento cognitivo progressivo, que leva à incapacidade funcional. Em 2015, estimou-se que aproximadamente 47 milhões de pessoas em todo o mundo foram afetadas pela demência, e esse número deve aumentar, atingindo 131,5 milhões em 2050 (PRINCE et al. 2015).

A doença de Alzheimer (DA), patologia descrita pela primeira vez por Alois Alzheimer em 1907, é a causa mais freqüente de demência em idosos. O conhecimento sobre a etiologia e patogênese da doença é continuamente atualizado (SPERLING et al 2011), mas, ainda, há limitações na capacidade diagnóstica e na descoberta de tratamentos farmacológicos, que poderiam impedir o avanço ou prevenir, de forma mais eficaz, a doença (LOPEZ et al 2010).

Atualmente, DA é incurável. Apesar da enorme quantidade de investigação pré-clínica e clínica, os medicamentos atualmente utilizados fornecem, apenas, alívio sintomático aos pacientes, deixando de tratar as causas subjacentes da doença. As razões para esta falha devemse, provavelmente, ao escasso conhecimento dos mecanismos celulares e moleculares implicados na patogénese da DA e das terapias aprovadas que afetam a neurotransmissão colinérgica e glutamatérgica. Por outro lado, os novos fármacos em desenvolvimento visam modificar o processo da doença em si, afetando uma ou mais, das muitas alterações cerebrais acometidas pela DA (BRONZUOLI et al 2016).

Fisiopatologicamente a DA é uma desordem multifatorial, caracterizada por um estresse oxidativo generalizado, danos mitocondriais, excitotoxicidade do glutamato, neuroinflamação, formação de emaranhado neurofibrilar (NFT) e deposição de $\beta$-amilóide (A $\beta$ ) criando placas senis (SPs) entre os neuronios, imposibilitando as sinapses (HENRY, W.; QUERFURTH, H. W.; LAFERLA, F. M, 2010).

As placas senis, são constituídas pelo péptido $A \beta$, e sua génese é seguida por deposições intracelular de NFTs, como consequência da hiperfosforilação da proteína tau. Os resultados são disfunções sináptica e neuronal. Ao longo dos anos, tem sido demonstrado que outros fatores desempenham um papel importante na patogênese e progressão da DA, entre eles, o papel da neuroinflamação (FERREIRA et al 2014).

O processo inflamatório tem como objetivo controlar as lesões através de vários mecanismos de reparação tisular e para eliminar as celulas mortas resultantes da lesão original. No entanto, uma quantidade crescente da literatura confirma seu papel na patogênese e exacerbação da DA (PROKOP, S.; MILLER, K. R.; HEPPNER, F. L., 2013.) 
Atualmente existem disponíveis biomarcadores para a DA que possibilitam a detecção do peptídeo $\beta$-amiloide ( $\beta \mathrm{A}-42)$ e da proteína tau, que apresentam uma correlação direta com a doença. Isto torna-se importante, vez que, o emprego desses biomarcadores realizados nos últimos anos evidenciaram que o processo fisiopatológico da DA pode ser identificado, tanto em indivíduos assintomáticos, como em pacientes com a demência instalada (FORLENZA, O. V.; DINIZ, B.S.; GATTAZ, W. F., 2010).

Segundo o Departamento de Neurologia Cognitiva e do Envelhecimento, da Academia Brasileira de Neurologia, foi elaborado os seguintes critérios para o diagnóstico da DA, no Brasil: início insidioso (meses ou anos); história clara ou observação de piora cognitiva; déficits cognitivos iniciais e mais proeminentes.

Para atingir o diagnóstico, conforme o estudo da Academia Brasileira de Neurologia, é necessário considerar as seguintes categorias: apresentação amnéstica; apresentação nãoamnéstica; linguagem (lembranças de palavras); visual-espacial (cognição espacial, agnosia para objetos ou faces, simultâneo agnosia, e alexia); funções executivas (alteração do raciocínio, julgamento e solução de problemas); tomografia ou, preferencialmente, ressonância magnética do crânio deve ser realizada para excluir outras possibilidades diagnósticas ou comorbidades, principalmente a doença vascular cerebral (FROTA F. et al. 2011).

Assim, a Doença Alzheimer, classifica-se em três fases principais:

- Fase inicial, que significa os estágios iniciais da doença, podendo durar de 0 a 4 anos, caracterizando-se pela perda da memória recente, como esquecimentos, falta de habilidade em aprender e reter novas informações, problemas na comunicação (linguagem), erros de julgamentos e progressiva dificuldade em realizar atividades diárias normais como ações de higienização. Nesta fase, os pacientes apresentam, geralmente, quadro clínico que dificulta o diagnóstico preciso.

- Fase intermediária, quando ocorre a progressão da doença, durando de 2 a 8 anos, a partir do diagnóstico. Nesta fase ocorrem perdas cognitivas adicionais; distúrbio na linguagem; repetição das palavras; interferência nas atividades ocupacionais; incapacidade de auto-cuidado; alto risco de acidentes secundários; agitação; falta de compreensão; e atitudes agressivas. O paciente necessita de acompanhamento constante, inicia-se, então, uma condição de semidependência em relação às atividades da vida diária. 
- Fase final, quando a doença está em seu estágio mais avançado, com duração de 6 a 10 anos. É determinada pela desorientação profunda; apatia; dependência total; incontinência fecal e urinária; perda completa da memória recente. Também pode ocorrer agressividade e ansiedade. Nesta fase é comum o risco de desnutrição. O progresso gradual leva o paciente ao coma e a morte (MIRANDA et al 2010).

A Doença de Alzheimer esta associada com uma variedade de sintomas não cognitivos, por exemplo, manifestações afetivas como: depressão e deteriorização da função motora. Assim, estes sintomas não cognitivos podem ser sinais precoces do desenvolvimento da DA (SCARMEAS, N. et al, 2005; WANG, L. et al, 2006).

Ainda, podem ser observadas em pacientes com DA: distúrbios de marcha (diminuição da velocidade da marcha, redução no comprimento do passo e redução na largura do passo); diminuição da força de membros superiores e inferiores; e alterações no controle postural. Tais distúrbios podem se apresentar na fase inicial da demência ou mesmo em estágios pré-clínicos da DA (SCHERDER, E. et al 2007).

Segundo Zidan et al. 2012, o maior declínio motor ocorre nas fases moderada e grave da DA, quando comparada com a fase leve. Apesar do declínio motor, cognitivo e da capacidade funcional, serem observados desde o início da DA, esse declínio não ocorre com a mesma magnitude nas diferentes fases da doença. A perda da independência dos pacientes em realizar as atividades básicas da vida diária (ABVD) não é consistente com o declínio cognitivo e físico observado nos testes neuropsicológicos e físicos.

Contudo, processos motores regulados por mecanismos corticais complexos (como sentar e levantar da cadeira) podem ser afetados na fase leve e moderada da DA, predispondo o paciente ao risco de quedas (KATO, M. et al 2005).

De fato, as quedas são mais frequentes e têm consequências traumáticas mais graves, incluindo fratura de quadril, em indivíduos com DA do que em idosos sem demência (GAGO M. et al 2016).

Burns et al 2010, associou que a perda de peso não intencional - que começa anos antes do reconhecimento dos sintomas clínicos -, relacionados à DA, pode ser um marcador de DA pré-clínica. A perda de peso na DA está associada a gravidade da demência e a progressão clínica mais rápida. A perda de massa magra é acelerada na DA, associando-se com atrofia 
cerebral e desempenho cognitivo, podendo ter consequência direta ou indireta na fisiopatologia da DA ou através de mecanismos compartilhados comuns tanto à DA como à sarcopenia.

Assim, a perda de força muscular em pessoas mais velhas com DA, pode levar a vários resultados negativos, como: limitações nas atividades de vida diária e quedas, resultando em lesões e mortalidade.

A habilidade para realizar as tarefas cotidianas envolve responsabilidade a ser compartilhada entre idosos, cuidadores e profissionais, por meio de cuidados coordenados e contínuos que proporcionem as condições básicas para um desempenho satisfatório.

As atividades cotidianas e funcionais são caracterizadas pelas atividades básicas de vida diária, as quais englobam a mobilidade básica (transferência, deambulação) e o cuidado de si (alimentação, higiene pessoal, vestir-se). Já as atividades instrumentais de vida diária (AIVD) se referem a um conjunto vasto de tarefas comuns que um indivíduo deve ser capaz de executar para manter sua independência, autonomia e relacionamento social, tais como: preparar alimentos, administrar o dinheiro, utilizar transporte e se medicar (CONDE-SALA, J. L. et al.2013).

Em uma pesquisa realizada na Bélgica, com 1289 pacientes com DA em estágio leve e moderado, foi constatado que $69 \%$ já necessitavam de mais de 12 horas de supervisão por dia, o que demonstra que, no início da evolução da doença, os pacientes apresentam-se bastante dependentes e com déficit da capacidade funcional (CARO et al, 2002).

Por outro lado, o idoso portador da DA na fase leve e moderada, consegue realizar as ABVD no ambiente domiciliar, mas não possui independência no ambiente externo para a realização das AIVD (LENARDT, M. H. et al. 2011).

Em um estudo transversal feito no Centro de Referência em Doença de Alzheimer do Município de Curitiba/PR, mostrou que grande parcela dos idosos (40\%) com demência de Alzheimer preservava uma ou mais ABVD no estágio inicial e moderado da doença, o que indica que essas atividades precisam ser valorizadas pelos cuidadores e profissionais, com o intuito de promover a autonomia e independência pelo maior tempo possível.

Concernente às AIVD, os escores revelaram que o idoso possui maior dificuldade na administração do dinheiro e em utilizar transporte, situações fundamentais para o convívio social. Estes fatores conduzem o idoso ao isolamento social e, consequentemente, à permanência em período integral no domicílio, necessitando de cuidados especiais. Assim, a presença de um cuidador torna-se fundamental. 
A avaliação do desempenho das ABVD e AIVD, viabiliza propostas de reabilitação eficazes e orienta as ações de cuidado dos profissionais da saúde e dos cuidadores dos idosos com DA, cujo principal objetivo é protelar os agravos da doença e favorecer melhor qualidade de vida para o portador e para o cuidador (MEDEIROS, M. E.; RO, G,2009).

Com isto, as intervenções destinadas a manter a independência em atividades básicas da vida diária (ABVD) em pessoas com DA, normalmente usam programas direcionados, tais como: treinamento funcional das ABVD; treinamento das atividades instrumentais da vida diária (AIVD); treinamento de atividades sociais e de lazer (LETTS et al, 2011).

\subsection{Equoterapia com a população idosa}

A Equoterapia é definida como um trabalho físico ou estratégia de tratamento, que utiliza o movimento do cavalo, geralmente como parte de um programa de tratamento integrado, para alcançar resultados funcionais. A Equoterapia é um importante método que fornece uma atividade prazerosa com exercício físico para indivíduos com deficiências no desenvolvimento e deficiências adquiridas ao longo da vida (HOMNICK, D. N. et al., 2013).

Esta forma de terapia, gera uma estimulação sensorial integrada, incluindo os sistemas vestibular, tátil, visual e postural, promovendo a estabilização da cabeça e do tronco, bem como uma reação motora geral. A Equoterapia engloba não só aquelas atividades baseadas no movimento do cavalo, mas também várias formas de postura e treinamento de movimentos para o cavaleiro, que são realizadas durante as sessões de terapia (SUNWOO, H. et al., 2012).

Assim, os cavaleiros são guiados em diferentes posições de montaria sobre o cavalo, a partir da posição tradicional de montaria (sentado para a frente), montaria de costas (sentado para trás em relação à cabeça do cavalo) ou sentado de forma lateral, com ambas pernas para um lado da sela. Às vezes, os cavaleiros são direcionados para adotar outras posições sobre o cavalo, como deitar de bruços, deitar de costas, ou posições em pé, dependendo do objetivo do tratamento (STRAUSS I., 1995).

O fundo teórico da Equoterapia é a aprendizagem e o controle motor, ou seja, um desempenho intensivo da experiência de aprender, baseado na reação ao movimento rítmico do cavalo. A caminhada do cavalo, em particular, consiste em movimentos precisos, suaves, rítmicos e repetitivos semelhantes à marcha humana. Por isso, repetidamente, fornece experiências semelhantes à caminhada para os sujeitos com declínio na função da marcha (MCGEE, M.C., REESE, N.B., 2009). 
O cavalo realiza em média 55 passos por minuto, à uma velocidade de marcha moderada, levando ao cavaleiro a executar entre 3.000 a 5.000 vezes o movimento de contrapeso do tronco ao montar o cavalo pelo período de 30 a 45 minutos (STRAUSS I., 1995).

Assim, a cada marcha do cavalo, deslocando o peso das patas traseiras da direita para esquerda, permite que a pelve do cavaleiro se mova, tanto verticalmente, como horizontalmente, junto aos três eixos de rotação do movimento (JANURA, M. et al., 2015).

Respostas neurosensoriais e neuromusculares são geradas a partir de grupos musculares utilizados pela estimulação mecânica. Embora ainda não definido, o movimento do cavalo também pode melhorar as respostas vestibulares para alterações na postura, levando à melhor proteção contra quedas de indivíduos mais velhos (HOMNICK, D. N. et al, 2013).

O conceito da Equoterapia na Europa central relaciona-se com os princípios neurofisiológicos da estimulação do desenvolvimento psicomotor e da ativação de programas de estimulação motora, podendo ser utilizado por pacientes com vários níveis de deficiência física (SVOBODA, Z. et al. 2011).

A Equoterapia é considerada método de tratamento e reabilitação há muito tempo, e atualmente vem sendo utilizada como técnica terapêutica para a melhora no controle do equilíbrio. Durante a Equoterapia, o paciente não só controla o equilíbrio através de movimentos ativos (voluntários), mas, também, promove o controle do equilíbrio (involuntário) como resposta aos movimentos do cavalo (DEBUSE, D., CHANDLER, C., GIBB C., 2005).

Segundo Kim S. e Lee CH., 2014, quando o cavaleiro é mediado pelo cavalo para controlar os tônus musculares anormais e os padrões de movimento, melhora-se o controle do equilíbrio do tronco e da marcha. Inferindo-se, assim, que a Equoterapia pode ajudar nas funções dos idosos, melhorando o equilíbrio e, portanto, prevenindo quedas. Os resultados do estudo feito por Kim e Lee avaliaram um grupo de idosos que praticava exercícios em esteira e outro grupo de idosos que fizeram Equoterapia. Concluíram que ambos os grupos obtiveram ganho no equilíbrio estático. Contudo, o grupo que praticou Equoterapia mostrou maiores efeitos.

Acredita-se que a diferença se deve ao fato de que a Equoterapia é composta de movimentos mais dinâmicos e em diferentes eixos de movimentos (para cima, para baixo, para a esquerda e direita e para a frente e para trás do cavalo) quando comparados com os exercícios em esteira, o que resulta em maior estímulo de respostas posturais dinâmicas.

Sung Y. et al., 2013, avaliaram 20 sujeitos com uma média de idade de 52 anos que sofreram AVC (isquêmico e hemorrágico), após 4 semanas de intervenção, investigaram se o 
uso do simulador de Equoterapia afetava a ativação dos músculos do tronco durante o movimento de sentar e levantar da cadeira. A ativação do eretor espinhal foi aumentada de $24,48 \pm 2,17$ para 24,54 $\pm 1,73$ no grupo controle (recebeu só fisioterapia convencional como tratamento) e foi significativamente aumentada de 23,97 $\pm 5,16$ para 29,07 \pm 5,98 no grupo experimental (recebeu fisioterapia convencional mais Equoterapia). Além disso, o grupo experimental apresentou diferença significativa, quando foi comparado com o grupo controle.

Em idosos saudáveis, que participaram de programa de Equoterapia de 8 semanas de duração, o tratamento foi efetivo para melhorar a força muscular nos membros inferiores (ARAÚJO, T.B. et al, 2013). Os efeitos da Equoterapia na função muscular dos idosos podem ser atribuídos à adaptações motoras, resultantes da combinação da estimulação sensorial proporcionada pelo cavalo. (MCGIBBON, N. H. et al 2009)

Pesquisa na base de dados (Pubmed, Medline, PEDRO), mostrou que para o período de 2000 a 2016, foram encontrados poucos estudos referente ao uso da Equoterapia em idosos. Os demais estudos tratam-se de pesquisas com foco em crianças com paralisia cerebral e pacientes com escleroses múltipla de diferentes idades. (ANTUNES et al, 2016; LINDROTH, J. L.; SULLIVAN, J. L.; SILKWOOD-SHERER, D, 2015). Não se encontra estudos sistemáticos sobre o potencial benefício da Equoterapia em indivíduos mais velhos com demência.

\subsection{Fisioterapia (Cinesioterapia funcional) em idosos com DA.}

Existem diversas estratégias de intervenção fisioterapêutica em indivíduos portadores de doença de Alzheimer. A intervenção fisioterapêutica pode ser realizada no domicílio do paciente ou em alguma clínica, e contribui, significativamente, em qualquer fase da doença de Alzheimer.

A realização de fisioterapia propicia, dentre outros, estabilidade do quadro e a melhora do desempenho funcional do indivíduo. Cabe destacar que sempre deve ser considerado os fatores epidemiológicos, fisiopatologia, etiologia, critérios diagnósticos e quadro clínico, para melhor definição de qual modalidade de intervenção fisioterapêutica deve ser realizada nos indivíduos portadores da doença (ELY, J., GRAVE, M., 2009).

A intervenção fisioterapêutica é intensamente recomendada para manter a amplitude de movimento, da força e do estado de alerta, também é importante para evitar atrofias por desuso e fraquezas musculares, os encurtamentos de tecidos moles e as deformidades esqueléticas (ZAIONS, 2012; MELO, 2006, KOTTKE; LEHMANN, 2002). 
A incapacidade funcional, decorrente da doença de Alzheimer, pode acarretar reforço da imobilidade e dos padrões de movimento estereotipados, podendo contribuir para o aparecimento de mudanças estruturais de grupos musculares, diminuindo a capacidade funcional (REBELATTO; MORELLI, 2004).

Evidências crescentes sugerem que a inatividade física é também um fator de risco potencial para o comprometimento cognitivo e demência em adultos mais velhos (ERICKSON, K.I.; WEINSTEIN, A. M.; LOPEZ, O. L, 2012).

Dentre as várias técnicas disponíveis, a cinesioterapia é a modalidade utilizada para manter ou melhorar a amplitude de movimento e a força muscular. Nas primeiras fases da doença um programa de alongamentos, de exercícios com carga e aeróbicos ajudam a prevenir problemas osteoarticulares e cardiovasculares. A cinesioterapia associada a hidroterapia e ao padrão respiratório são essenciais, uma vez que a capacidade funcional da fala, respiração, expansão torácica e função venosa, vão diminuindo aos poucos nos portadores da Doença de Alzheimer (MENDONÇA, 2011; DE CARVALHO et al.,2009; ELY, 2009).

Nesse sentido, em 2006, foi criado o "Programa de Cinesioterapia Funcional e Cognitiva em Idosos com Doença de Alzheimer" (PRO-CDA), projeto de Extensão do Departamento de Educação Física da UNESP - Universidade Estadual Paulista, que tem por objetivo proporcionar aos pacientes participantes, os benefícios encontrados através da prática regular de atividade física (AF), buscando atenuar os sintomas progressivos da doença, contribuindo, assim, para melhor a qualidade de vida, tanto para pacientes, quanto para cuidadores (GARUFFI M., et al 2012).

As atividades do grupo são realizadas três vezes por semanas, em dias não consecutivos, com duração de 60 minutos cada sessão. Atualmente são realizados três protocolos de intervenção distintos: Convívio Social, Treinamento com pesos e Atividade Física para cuidadores.

O protocolo "Treinamento com pesos" é direcionado para os pacientes com demência de Alzheimer tem por objetivo promover melhorias na capacidade física, cognição e nos distúrbios neuropsiquiátricos dos participantes, além de evitar declínios decorrentes do avanço da doença. Em idosos cognitivamente preservados os benefícios do treinamento com pesos já estão comprovados (AMERICAN COLLEGE OF SPORTS MEDICEN, 2009). Contudo, pouco se sabe a respeito do treinamento com pesos em idosos com DA, sobretudo no campo da cognição e funcionalidade motora. 
Assim, a pratica de atividade física regular pode atenuar a atrofia cerebral, uma vez que ambas estão positivamente associadas ao volume da matéria cinzenta em adultos mais velhos (ERICKSON, K I.; LECKIE, R. L.; WEINSTEIN, A. M. 2014) e adultos mais velhos em risco de DA (OKONKWO, O. C. et al, 2014).

O Departamento de Saúde e Serviços Humanos, juntamente com o Colégio Americano de Medicina Esportiva e da Associação Americana de Cardiologia, recomendam a prática de 150 minutos de exercício físico de intensidade moderada, ou 75 minutos de exercícios de alta intensidade, ou uma combinação de ambas, por semana, para promover a saúde em todos os adultos (DOUGHERTY et al 2016).

Dadas as limitadas opções de tratamento, deve-se dar maior ênfase à prevenção primária através da atividade física entre indivíduos com alto risco de DA e com aqueles com forte histórico genético e familiar (BECKETT, M. W.; ARDERN, C. I.; ROTONDI, M. A., 2015).

Assim, as intervenções fisioterapêuticas sobre o déficit musculoesquelético na DA deve estar focalizada não somente sobre o treino do padrão da marcha e da manutenção da independência nas atividades da vida diária (REBELATTO; MORELLI, 2004), mas, também, no ganho de massa e força muscular.

Boyle menciona que correlacionou a força muscular de idosos sem DA, durante 3.6 anos, e concluiu que para uma unidade no incremento da força muscular (avaliados em 11 grupos musculares) diminuía-se, em 43\%, o risco de desenvolver DA (BOYLE et al, 2009).

A literatura têm mostrado a relação inversa entre força muscular e prevalência de DA. Por exemplo, após o transcurso de 5,5 anos, de um total de 900 idosos sem demência, 151 idosos, desenvolveram e foram clinicamente diagnosticados com DA. A incidência de DA nesse estudo teve forte associação com a baixa força muscular, avaliada com dinamômetro de preensão manual (BUCHMAN, A. S. et al.,2005).

Em outro estudo feito no México, com 91 idosos com idade média de 64 anos, mostrouse que a força de preensão manual também foi associada ao declínio cognitivo após um o decurso de 7 anos, mesmo após o controle de outros fatores potencialmente influenciadores de declínio cognitivo. Por outro lado, os sujeitos que apresentaram maior força na avaliação, mantiveram maior nível de função cognitiva (ALFARO-ACHA, A. et al., 2006). Contudo, a força de preensão manual não é o melhor indicador da força corporal total ou da medida do grupo muscular mais importante, em adultos mais velhos (HURLEY, B. F.; HANSON, E. D.; SHEAFF, A. K., 2011). 
A reabilitação é um processo contínuo de educação e de solução de problemas, e o objetivo da intervenção fisioterapêutica é a redução da incapacidade e da deficiência vivenciada por alguém. O processo de reabilitação também inclui a realização de modificações ambientais necessárias para a segurança do paciente, de modo que possa viver em um ambiente mais confortável (REBELATTO; MORELLI, 2004).

Assim, a abordagem fisioterapêutica tem papel muito importante na manutenção e na melhora do desempenho funcional, social e cognitivo do indivíduo portador da doença. É importante ressaltar que este fator pode contribuir diretamente na qualidade de vida destes indivíduos. 


\section{Metodologia}

\subsection{Tipo de Estudo}

O presente estudo é classificado como ensaio clínico quase-experimental.

\subsection{Amostra}

Participaram do estudo 60 idosos de ambos gêneros, dos quais 17 sujeitos cumpriram os critérios de inclusão e 43 estavam dentro dos critérios de exclusão, assim por conveniência foram divididos em Grupo Equoterapia (6 homens e 4 mulheres) e Grupo Fisioterapia (2 homens e 5 mulheres), com idade acima de 65 anos, com diagnóstico de Demência de Alzheimer fase leve e moderada, recrutados a partir de triagens que foram realizados no Centro de Medicina do Idoso (CMI), no Hospital Universitário de Brasília (HUB) no Distrito Federal (DF), no período de Janeiro-Abril de 2015.

Para participar do estudo foram estabelecidos como critérios de inclusão: a idade mínima de 65 anos, apresentar diagnóstico de Doença de Alzheimer fase leve e moderada, estar residindo no DF e apresentar atestado médico de liberação para a prática de Equoterapia. Foram excluídos da pesquisa idosos que apresentaram fobia ao cavalo, patologias traumatoortopédicas, visuais severas, auditivas graves, neurodegenerativas graves, reumatológicas, metabólicas ou cardiovasculares sem controle, hipertensão arterial sem controle, artroplastia de quadril ou joelho, presença de material de osteosíntese em articulações, escolioses severa, cirúrgia traumato-ortopédica nos últimos seis meses, fratura óssea ou lesão muscular nos últimos seis meses.

Aqueles indivíduos considerados aptos na triagem e que aceitaram participar da pesquisa, assinaram o Termo de Consentimento Livre e Esclarecido (TCLE), junto a aprovação do Comitê de Ética (parecer $\mathrm{N}^{\circ} 949.732$, tendo como pesquisadora responsável a Doutoranda Thais Borges de Araújo). 
O fluxograma apresentado na Figura 1 mostra o delineamento do estudo.

Figura 1- Delineamento do estudo

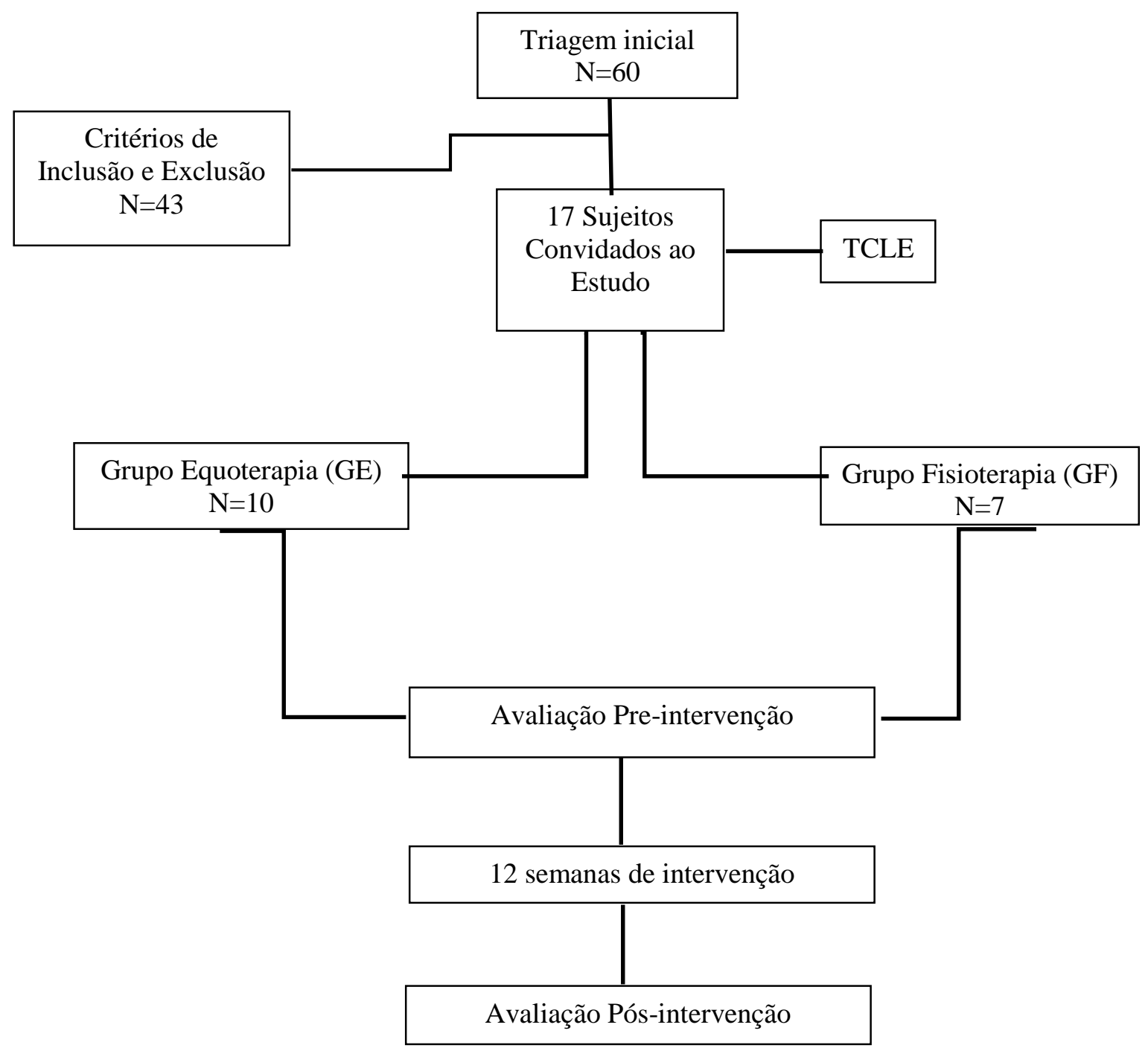

Fonte: Elaboração própria 


\subsection{Instrumentos e testes}

A avaliação do pico de torque (extensão e flexão de joelho) foi realizada em um dinamômetro isocinético (Biodex System ${ }^{\circledR}$, modelo III). Para avaliação das atividades da vida diária foi utilizada a Escala de Barthel, validada no Brasil (MINOSSO, J. S. M. et al.,2010), para a avaliação do estado cognitivo foi utilizado o Minimental Teste (MMSE).

Os procedimentos de avaliação obedeceram a mesma ordem de execução para ambos grupos, no momento Pré-intervenção (PRÉ) e após 12 semanas de intervenção (PÓS), da seguinte forma: $1^{\circ}$ MMSE, $2^{\circ}$ Indice de Barthel, $3^{\circ}$ Antropometria; $4^{\circ}$ Dinamômetria Isocinética.

A primeira semana do estudo foi dedicada à avaliação PRÉ dos sujeitos para os dois grupos de pesquisa. Seguiram-se 12 semanas de intervenção, tanto para o GE como para o GF. Para a avaliação PÓS foi respeitado, para o GE e GF, o intervalo mínimo de quatro dias após a última sessão de Equoterapia e Fisioterapia.

\subsection{Caracterização da Amostra}

\subsubsection{Avaliação do estado cognitivo}

O Nível do estado cognitivo, foi obtido por meio do Minimental Teste (MMSE) Foi desenvolvido por Marshal F. Folstein, Susan Folstein, and Paul R. McHugh em 1975, como um método para estabelecer o estado cognitivo do paciente e poder detectar demência.

Já foi validado para a população brasileira. Fornece informações sobre diferentes parâmetros cognitivos, contendo questões agrupadas em sete categorias, cada uma delas planejada com o objetivo de avaliar "funções" cognitivas específicas como a orientação temporal, orientação espacial, registro de três palavras, atenção e cálculo, recordação das três palavras, linguagem e capacidade construtiva visual. O escore do MMSE pode variar de um mínimo de 0 pontos, o qual indica o maior grau de comprometimento cognitivo dos indivíduos, até um total máximo de 30 pontos, o qual, por sua vez, corresponde a melhor capacidade cognitiva.

É uma ferramenta de avaliação rápida que permite suspeitar déficit cognitivo. Porém não permite detalhar o domínio alterado nem conhecer a causa da doença, portanto, nunca deve ser utilizado em substituição de uma avaliação clínica completa do estado mental. 


\subsubsection{Atividades básicas da vida diária (ABVD)}

Foram avaliadas pelo índice de Barthel, no caso das ABVD englobam a mobilidade básica (transferência, deambulação) e o cuidado de si (alimentação, higiene pessoal, vestir-se). A escala de Barthel (Anexo 4) avalia seis atividades (tomar banho, vestir-se, usar o banheiro, transferência, continência urinária/fecal, e alimentar-se) e o resultado pode variar entre zero e dez pontos, sendo que o valor mínimo (zero) significa que o indivíduo é dependente para as ABVD e o máximo (dez) que é independente. O teste foi aplicado aos cuidadores formais ou informais dos sujeitos avaliados, as entrevistas foram feitas pelo mesmo avaliador durante a realização da bateria de testes pré e pós intervenção, em uma sala isolada e registrados em folha de avaliação previamente delineada.

\subsubsection{Antropometria}

A antropometria foi coletada como parte do protocolo da caracterização dos sujeitos no dinamômetro isocinético, para isso usou-se uma balança eletrônica digital (Filizola®,modelo PersonalLine) com resolução de 100 gramas e um estadiômetro (Country Tecnology®, modelo 67031) com resolução de um centímetro.

\subsubsection{Força muscular isocinética}

A força muscular isocinética de extensão e flexão do joelho foi aferida pelo pico de torque (PT) nos membros dominantes, na velocidade de $60 \%$, segundo protocolo adaptado proposto por Bottaro, M.; Russo, A e Oliveira, R. J (2005). O membro inferior dominante foi definido pela preferência de um lado em chutar uma bola (HARTMANN, A. et al, 2008) O protocolo de aferição do PT no momento PRÉ e PÓS foi realizado da seguinte maneira: $1^{\circ}$ uma série de aquecimento de cinco repetições de extensão e flexão do joelho dominante a $300^{\circ}$ s; $2^{\circ}$ - duas séries de cinco repetições a $180 \%$ s de extensão e flexão do joelho dominante; $3^{\circ}$ - duas séries de cinco repetições a $60^{\circ}$ s de extensão e flexão do joelho dominante. O intervalo entre as séries de contrações foi de um minuto. 


\subsection{Procedimentos Experimentais}

Após o período de triagem, os voluntários foram alocados por conveniência, em dois grupos: Grupo Equoterapia (GE - n = 10) e Grupo Fisioterapia (GF - n =7). O período de treinamento do GE iniciou-se com uma semana para a familiarização com o Cavalo, seguida de 12 semanas do programa de Equoterapia (PE), sendo duas vezes por semana, com duração de 30 minutos por sessão. Durante a familiarização e treinamento do GE, utilizaram-se exercícios de aquecimento e alongamentos para os membros inferiores e membros superiores, mobilidade articular para articulações dos membros superiores e inferiores, executados sempre de modo alternado e com 10 a 20 segundos de intervalo de recuperação.

Os exercícios para os membros inferiores foram: a montaria clássica (sentado na sela), montaria esporte isométrica (desencostado da sela). Exercícios executados mantendo a posição por 10 segundos com 10 segundos de descanso. Sentar e levantar da sela, 4 series de 10 repetições, executados em diferentes momentos da sessão. Durante a sessão de Equoterapia, também foram executados outros exercícios, como comando de rédea (segurando a mesma ou puxando em diferentes direções) e exercícios com materiais didáticos (bolas, bambolês, elásticos). Estes exercícios foram executados com o cavalo parado, ao passo e ao trote. A Figura 2 demonstra a execução de exercícios do GE propostos pelo presente estudo.

Os exercícios do GF eram realizados 2 vezes por semana com 60 minutos de duração cada sessão. Os equipamentos utilizados eram halteres, caneleiras de $1 \mathrm{~kg}, 2 \mathrm{Kg}, 3 \mathrm{Kg}$ e $4 \mathrm{Kg}$, elastos de diferente intensidades e diferentes superfícies instáveis (disco de freeman, bolas, tapetes, espuma). A intensidade do exercício era controlada de acordo com a percepção subjetiva de esforço, através, da escala de Berg. A sessão de Fisioterapia, foi realizada no Hospital Universitário de Brasilia, no ambulatório do Centro de Medicina do Idoso. As atividades eram desenvolvidas com o apoio de cadeiras. Para os membros inferiores eram realizadas 4 séries de 10 repetições de extensão de joelho (sentado numa cadeira) e flexão de joelho em pé (apoiado numa cadeira). 3 séries de 10 repetições de sentar e levantar da cadeira com os braços cruzados no peito, 4 séries de 10 repetições de flexão plantar em pé bilateral, exercícios para os membros superiores com halteres e elastos tendo que completar 4 series de 10 repetições para bíceps, tríceps, peitoral e deltoides, além de exercícios proprioceptivos sobre diferentes superfícies instáveis. Na Figura 3, se mostra o GC recebendo a intervenção específica.

Durante o período do experimento, todos os voluntários foram orientados a não iniciarem qualquer outra atividade física e a não modificarem suas rotinas e hábitos alimentares. 
No primeiro dia do treinamento, todos os voluntários do GE foram orientados sobre possíveis eventos adversos decorrentes da prática da Equoterapia, detalhados no TCLE, sendo indagados em cada sessão sobre o surgimento de sintomas relacionados ao treinamento.

Toda a intervenção foi supervisionada por um Professor de Educação Física e um Fisioterapeuta.

Figura 2- Programa Equoterapia

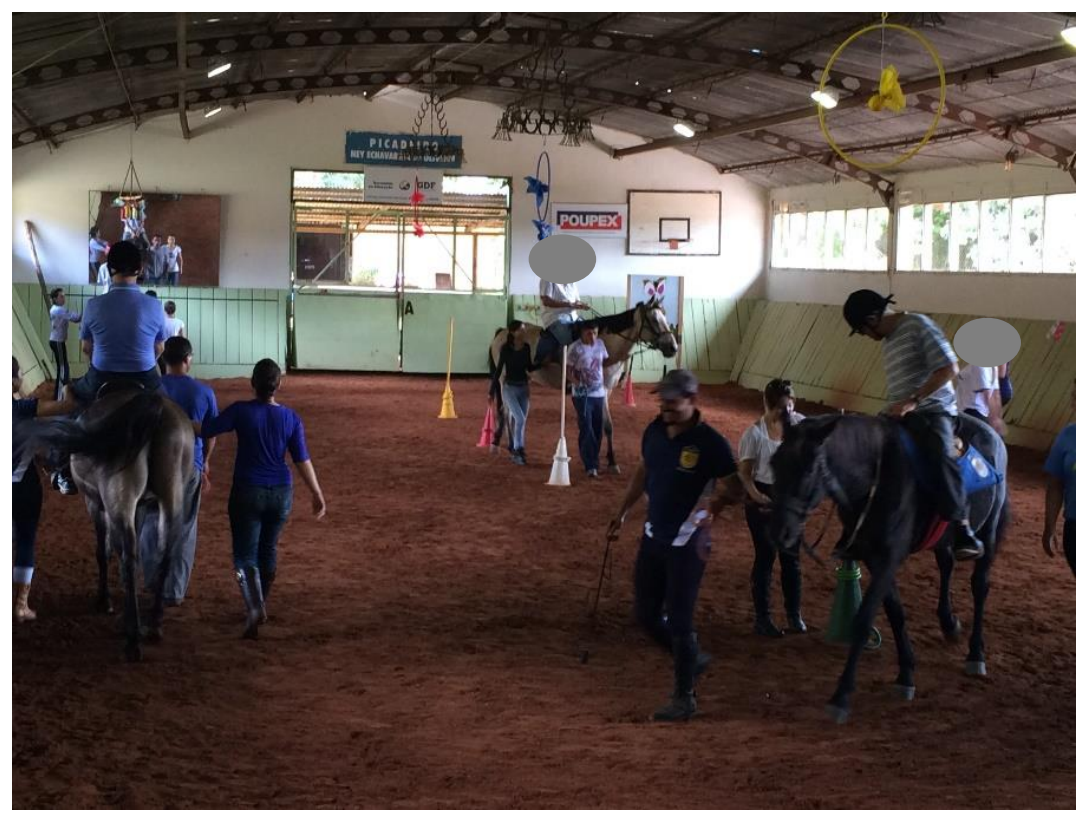

Fonte: Foto realizada pelo autor 
Figura 3. Programa Fisioterapia

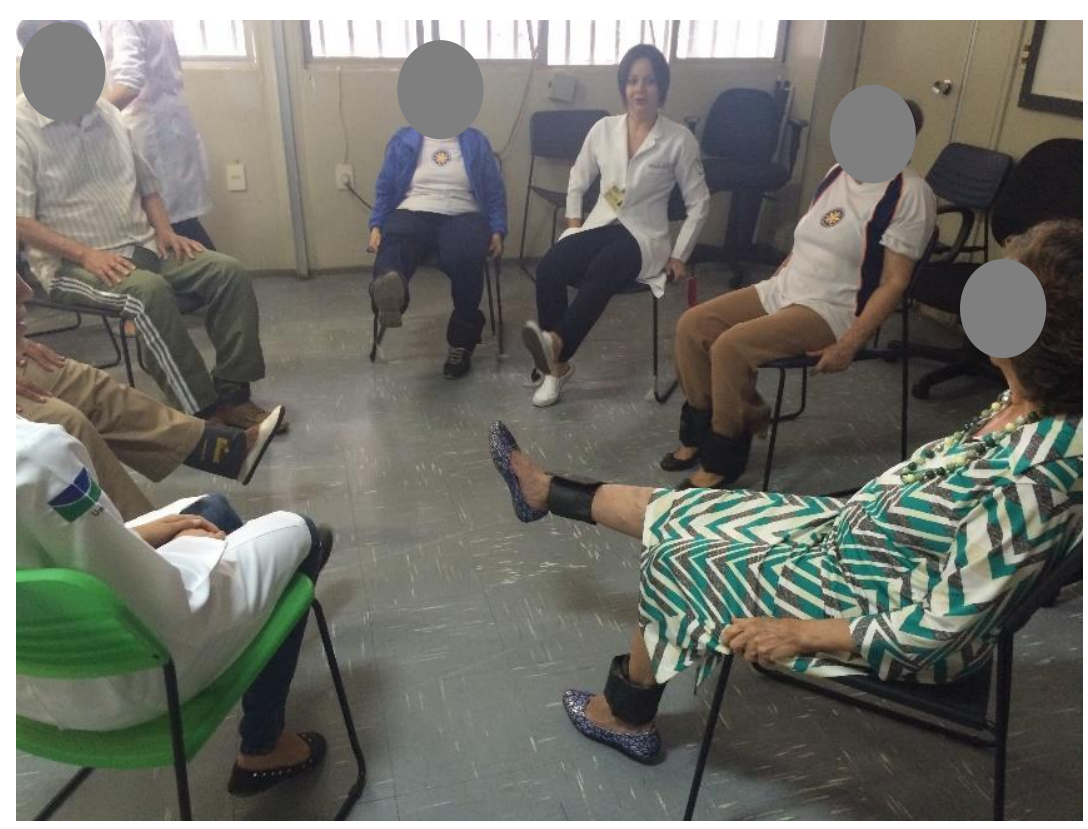

Fonte: Foto realizada pelo autor 


\subsection{Análise Estatística}

Foi realizada análise exploratória dos dados, com medidas descritivas de média \pm desviopadrão para organizar e apresentar os resultados. O estudo analisou as seguintes variáveis: pico de torque na flexão e na extensão do joelho. A normalidade das variáveis quantitativas foi confirmada pelo teste de Kolmogorov-Smirnov.

Para testar os efeitos do tipo de tratamento (PF ou PE) nas variáveis dependentes (PT), foi realizado um teste de Anova Splitplot (análise de variânza mista para medidas repetidas). Os efeitos das intervenções na escala de Barthel no que se refere ao nível de dependência funcional foi efetuado por meio do teste McNemar.

Para estatística descritiva foi utilizada a média e o desvio padrão, pelo teste $T$-student.Todas as análises foram realizadas no programa SPSS 17.0, adotando-se para os testes o nível de significância de $\mathrm{p} \leq 0,05$. 


\section{Resultados}

\subsection{Análise descritiva: perfil da amostra}

Dezessete participantes começaram e finalizaram a pesquisa, não se registrando perda amostral, por serem tratamentos dependentes de frequência de comparecimento, o que determinou a adesão aos programas. No GF participaram cinco sujeitos de sexo feminino e dois do sexo masculino, dos quais a mesma proporção eram independentes ou levemente dependentes, em relação às ABVD.

No GE participaram quatro sujeitos do sexo feminino e seis do sexo masculino, dos quais seis eram independentes e quatro levemente independentes, segundo o índice de Barthel. No que acomete ao estado cognitivo, os dezessete tinham demência, suas características Pré intervenção estão descritas na tabela seguinte:

Tabela 1- Estatísticas descritivas Pré dentro dos GF e GE

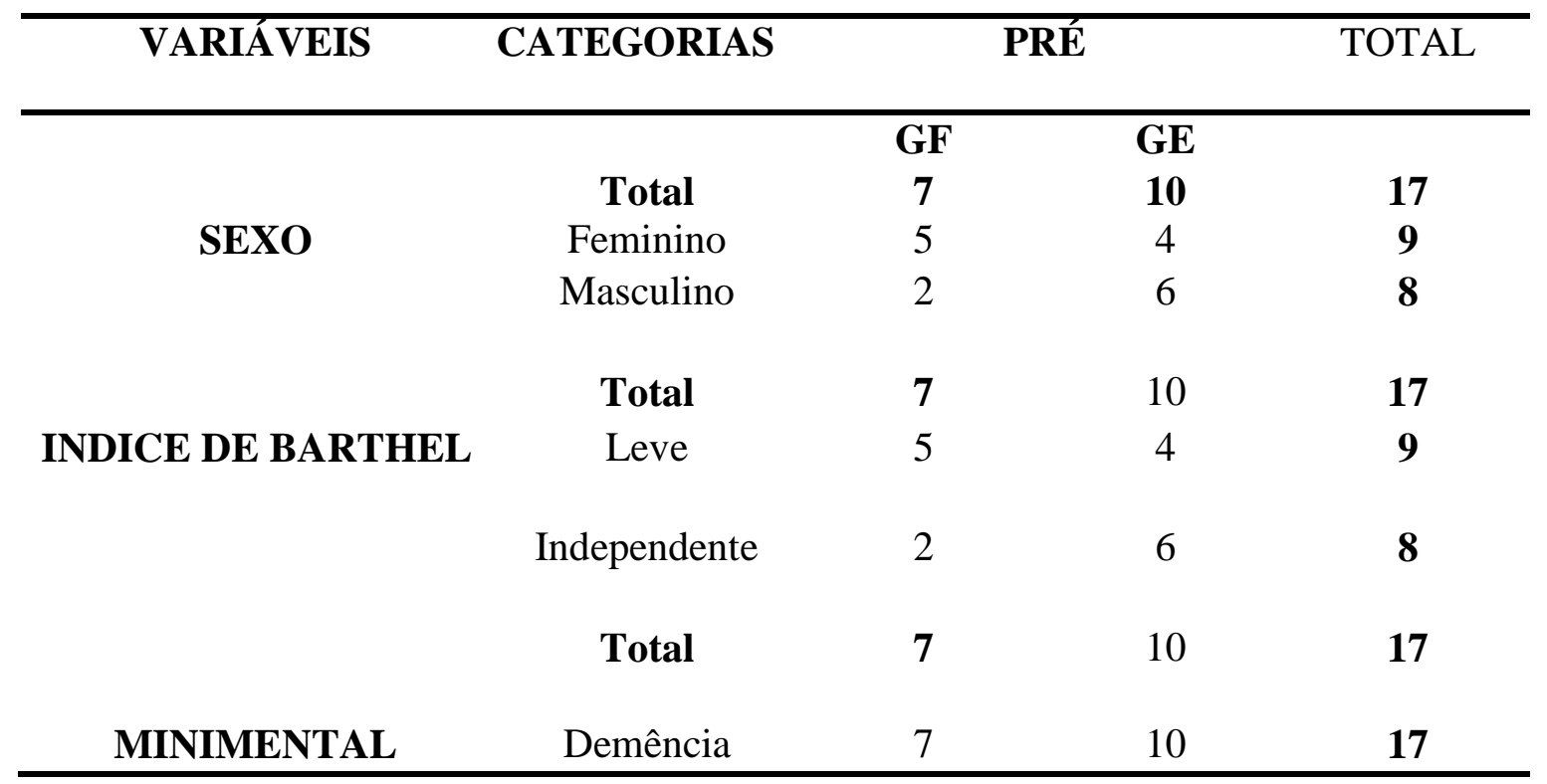

Fonte: Elaborada pelo autor 
A Tabela 2, apresenta a média e desvio padrão dos grupos nos momentos Pré e Pós das interveções. A idade dos participantes do estudo variou de 69 a 79 anos e teve uma média de 76,1 anos, com um desvio padrão de 2,3 anos, para o GF e de 74,4 com um desvio padrão de 3,3 anos para o GE. Constatou-se que para a variavel pico de torque na flexão do joelho (Flex 60 N-m), o GF apresentou uma diferencia de médias nos momentos Pré e Pós de 20,30\%, em quanto o GE, apresentou uma diferencia de 19,19\%, inferindose que ambos grupos apresentaram ganhos após as 12 semanas de intervenção.

Tabela 2- Média \pm Desvio Padrão - Pré X Pós Dentro Dos Grupos

\begin{tabular}{ccccccc}
\hline Variáveis & \multicolumn{3}{c}{ Grupo Fisioterapia } & \multicolumn{3}{c}{ Grupo Equoterapia } \\
& Pré & Pós & $\boldsymbol{\Delta} \%$ & Pré & Pós & $\boldsymbol{\Delta} \%$ \\
Idade & $76,1 \pm 2,3$ & $76,1 \pm 2,3$ & $0,00 \%$ & $74,4 \pm 3,3$ & $74,3 \pm 3,3$ & $0,00 \%$ \\
MMSE & $21,7 \pm 2,2$ & $21,7 \pm 2,2$ & $0,00 \%$ & $21,1 \pm 2,6$ & $21,1 \pm 2,6$ & $0,00 \%$ \\
Flex 60 (N-m) & $28,1 \pm 11,9$ & $33,9 \pm 13,2$ & $20,30 \%$ & $34,4 \pm 18,2$ & $41 \pm 21$ & $19,19 \%$ \\
Ext 60 (N-m) & $74,9 \pm 34,3$ & $72,7 \pm 39,7$ & $-2,86 \%$ & $88,9 \pm 41$ & $90,4 \pm 36,1$ & $1,69 \%$ \\
Indice Barthel & $92,1 \pm 8,6$ & $92,9 \pm 9,1$ & $0,78 \%$ & $93,5 \pm 11,8$ & $92 \pm 9,8$ & $-1,60 \%$ \\
& & & & & & \\
\hline
\end{tabular}

MMSE: minimental teste, $\Delta \%$ : diferencia de médias

Fonte: Elaborada pelo autor 
De acordo com a Tabela 3, Nos resultados da ANOVA mista (Split-plot), observou-se um efeito principal intra grupos GF e GE, na variavel dependente pico de torque de flexão de joelho (60 N-m), no momento Pré-Pós $(\mathrm{F}=19,26, \mathrm{p}=0,001)$. Entretanto, não houve interação significativa entre os grupos para a mesma variavel nos momentos Pré-Pós intervenção $(\mathrm{F}=$ 0,145, $\mathrm{p}=0,708)$. Em ambos os grupos, o efeito do teste entre sujietos, indicou que não havia diferença significativa entre os tratamento $(F=0,622, p=0,443)$.

Tabela 3-Teste Anova (Split-plot) para amostras pareadas - Pré x Pós dentro dos grupos

\begin{tabular}{ccc}
\hline EFEITO & F & P-valor \\
\hline Pré-Pós Flex 60 Nm & $\mathbf{1 9 , 2 6}$ & $\mathbf{0 , 0 0 1}$ \\
Pré-Pós Flex * Tratamentos & 0,145 & 0,708 \\
Tratamentos & 0,622 & 0,443 \\
\hline
\end{tabular}

Fonte: Elaborada pelo autor 
O gráfico 1 apresenta o comportamento da variável dependente pico de torque na flexão de joelho nos momentos Pré-Pós intervenção, para os grupos submetidos a Fisioterapia e a Equoterapia. Note-se que para ambos os grupos houve ganho de força. No grupo submetido ao tratamento de Equoterapia encontrou-se, ainda, ganho de força muscular no momento Pós intervenção, maior que o grupo que foi submetido aos tratamento de Fisioterapia.

Gráfico 1-Estimação de Médias Marginais para flexão de joelho

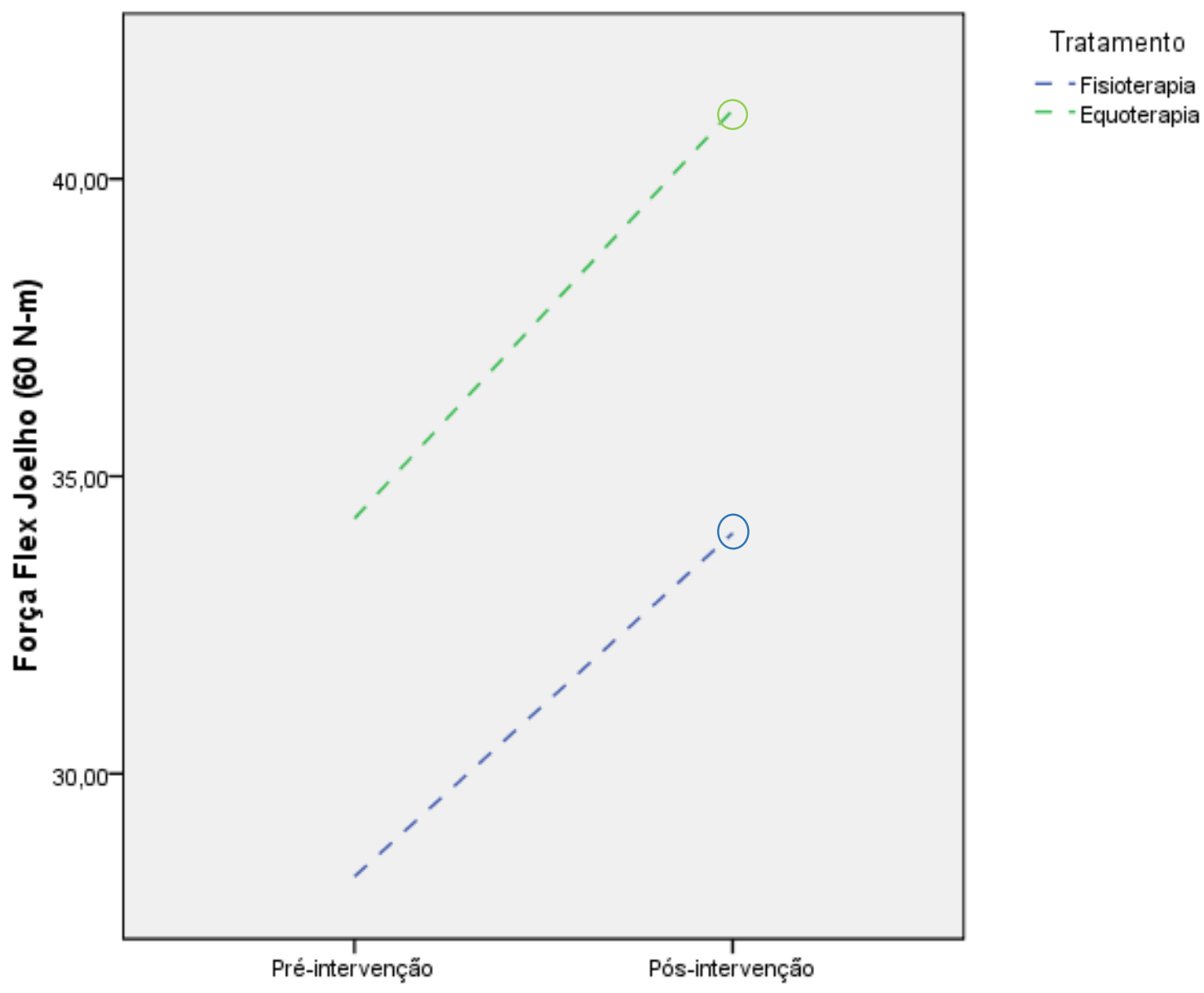

Fonte: Elaborada pelo autor 
De acordo com a Tabela 4, Nos resultados da ANOVA mista (Split-plot), observou-se um efeito principal intra grupos GF e GE, na variavel dependente pico de torque de extensão de joelho (60 N-m), no momento Pré-Pós $(\mathrm{F}=0,019, \mathrm{p}=0,893)$, não havendo diferencias estatisticamente significativas. Entretanto, não houve interação significativa entre os grupos para a mesma variavel nos momentos Pré-Pós intervenção $(F=0,400, p=0,537)$. Em ambos os grupos, o efeito do teste entre sujietos, indicou que não havia diferença significativa entre os tratamento $(\mathrm{F}=0,722, \mathrm{p}=0,409)$.

Tabela 4-Teste Anova (Split-plot) para amostras pareadas - Pré x Pós dentro dos grupos

\begin{tabular}{ccc}
\hline EFEITOS & F & P-valor \\
\hline Pré-Pós Ext 60 Nm & 0,019 & 0,893 \\
Pré-Pós Ext * Tratamentos & 0,400 & 0,537 \\
Tratamentos & 0,722 & 0,409 \\
\hline
\end{tabular}


O gráfico 2, apresenta o comportamento da variável dependente pico de torque na extensão de joelho nos momentos Pré-Pós intervenção, para os grupos submetidos a Fisioterapia e a Equoterapia. Note-se que o comportamento dos grupos foi diferente, houve um ligeiro ganho de força no GE, embora não chegou a ser estatisticamente significativo. No grupo submetido ao tratamento de Fisioterapia encontrou-se, ainda, uma ligeira queda na força muscular no momento Pós intervenção.

Gráfico 2-Estimação de Médias Marginais para extensão de joelho

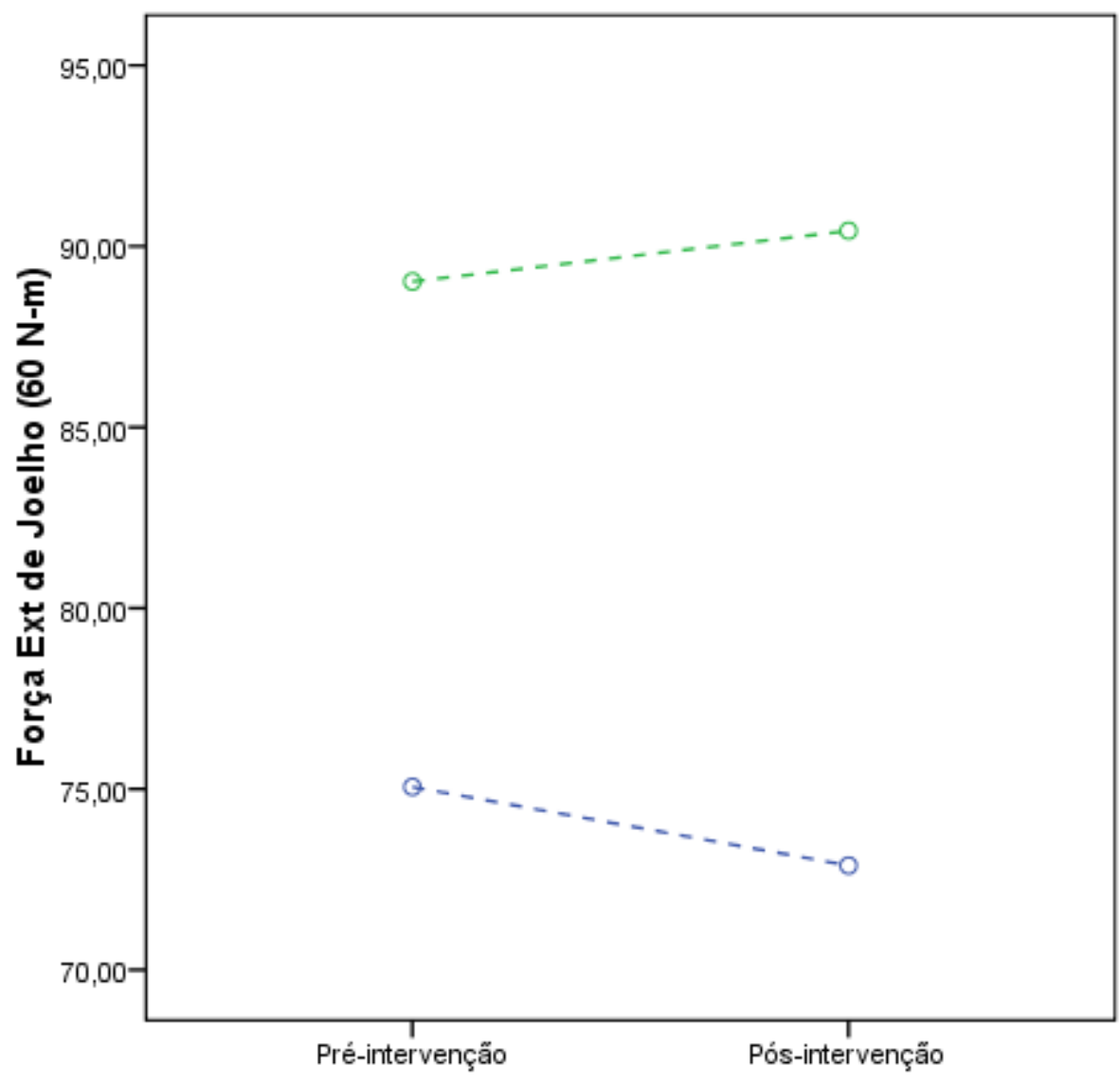

$$
\begin{gathered}
\text { Tratamento } \\
-- \text { Fisio } \\
-- \text { Equo }
\end{gathered}
$$

Fonte: Elaborada pelo autor 
A Tabela 5 apresenta os efeitos da Equoterapia e da Fisioterapia Convencional no índice de Barthel no que refere ao nível de dependência funcional dos voluntários. Notavelmente, não houve diferença significativa entre os momentos pré e pós-intervenção em nenhuma das modalidades estudadas $(p=1,000)$.

Tabela 5-Teste McNemar para o efeito da Equoterapia e da Fisioterapia convencional no nível de dependência funcional. Dados expressos em frequência absoluta e relativa.

\begin{tabular}{lccc}
\hline & $\begin{array}{c}\text { Pré } \\
\mathrm{n}(\%)\end{array}$ & $\begin{array}{c}\text { Pós } \\
\mathrm{n}(\%)\end{array}$ & $P$ \\
\hline Grupo Equoterapia $(\mathrm{n}=10)$ & & & \\
$\quad$ Dependência leve & $4(40,0)$ & $5(50,0)$ & 1,000 \\
$\quad$ Independência & $6(60,0)$ & $5(50,0)$ & \\
Grupo Fisioterapia $(\mathrm{n}=7)$ & & & \\
$\quad$ Dependência leve & $5(71,4)$ & $4(57,1)$ & 1,000 \\
Independência & $2(28,6)$ & $3(42.9)$ & \\
\hline
\end{tabular}

Fonte: Elaborada pelo autor 


\section{Discussão}

O objetivo do presente estudo foi analisar e comparar os efeitos do programa de Equoterapia e do programa de Fisioterapia Convencional, pelo período de doze semanas, na força muscular dos membros inferiores de idosos com Doença de Alzheimer na fase leve e na fase moderada. Além disso, foram analisados os efeitos Pré e Pós-intervenção da Equoterapia e Fisioterapia sobre o pico de torque do joelho dominante de idosos com DA, nas fases leve e moderada. Os resultados mostraram que houveram diferenças significativas no pico de torque na flexão de joelho dos idosos, quando submetidos aos programas de Equoterapia e Fisioterapia Convencional. Já em relação ao pico de torque na extensão de joelho, não houve diferença estatisticamente significativa no momento Pós-intervenção.

Diante dos principais achados do presente estudo, constatou-se um ganho na produção de força, na flexão do joelho $(\mathrm{p}=0,001)$ para ambos grupos, tendo uma diferença estatisticamente significativa ( $\mathrm{p}$-valor < 0,05), apenas no pico de torque da flexão do joelho (Flex $60 \mathrm{~N}-\mathrm{m}$ ).

Estes resultados concordam com os descritos em estudo anterior, realizado por Bento $\mathrm{P}$. et al. em 2010, quando analisaram o desenvolvimento do pico de torque de diferentes grupos musculares do membro inferior, em idosos que tinham média de idade de 67 anos e que foram divididos em 3 grupos ( $\mathrm{G} 1=$ sem queda, $\mathrm{G} 2=$ uma queda, $\mathrm{G} 3=$ duas ou mais quedas).

Os idosos estudados por Bento P no 2010, apresentavam episódios de queda durante 12 meses, e, segundo sua análise, o único grupo muscular que apresentou diferença estatisticamente significativa nos flexores de joelho $(\mathrm{p}=0,002)$, foram os idosos que não apresentavam quedas. Assim a maior taxa de desenvolvimento de torque dos músculos flexores do joelho, foi encontrada nos idosos que não sofreram quedas, em comparação com os idosos que sofreram quedas.

Os achados do presente estudo tornam importantes do ponto de vista da funcionalidade dos sujeitos, tedno em vista o fato da doença de Alzheimer constituir uma doença progresiva e irreversível, possibilitando, com os ganhos da força muscular nos extensores dos joelhos, o retardo ou freio no tão acelerado processo degenerativo.

Na metanalise realizado por Peterson M.D. et al. 2010, constatou-se uma associação robusta e significativa entre o exercício de resistência e a melhoria da força corporal superior e inferior entre indivíduos mais velhos. Assim, é alta a frequiência com que a força de extensão do joelho é relatada na literatura, bem como a relevância da força de membros inferiores para a locomoção, para as atividades da vida diária e para o risco de quedas. Assim, os resultados do presente estudo diferem, pois, no pico de torque da extensão de joelho não se apresentou ganhos 
estatisticamente significativos. Tal situação pode se dar pelo fato dos estudos inclusos na metanalises, ter analisado diferentes tipos de intensidades, duração e volume, com frequencias de treinos maiores.

No estudo clínico randomizado de Gadelha B. A. et al. em 2016, foi avaliado o efeito do treinamento resistido em idosas, e constatou-se alterações nos valores de 1RM após 24 semanas para o GE, sendo esses valores: 78\%, 54\%, 42\%, 34\%, 70\% e 33\% para Leg press, extensão do joelho, flexores de joelho, abdução de quadril, supino e lat pulldown respectivamente. Todos os valores aumentaram significativamente $(\mathrm{p}<0,01)$ para todos os exercícios avaliados. O que concorda com o presente estudo, que aferiu que o incremento na força dos flexores de joelho aumentou 20,3\%, após 12 semanas de intervenção para o GF e 19,2\% para o GE.

Bottaro et al em 2007, avaliou os efeitos de 10 semanas de um programa de treinamento de força versus um programa tradicional de treinamento resistido, em 20 idosos do sexo masculino. Para a avaliação da força muscular de membros inferiores, na pré e na pós intervenção, foi utilizado o teste de 1RM, dinamometro isocinetico e o teste de sentar e levantar da cadeira. Ambos programas de intervenção foram efetivos na melhora da força de membros inferiores. No entanto, o treinamento de força foi mais efetivo que o treinamento tradicional de exercicio resistido. Apresentando novamente uma correlação com o presente estudo, aonde se obtive ganho nos dois programas de intervenção, sendo mais efetivo o progama de Equoterapia, podendo ser considerado um treinamento de força, uma vez que foram realizados exercicios acima dos estribos e com o cavalo em movimento, exigendo diferentes velocidades de execução e diferentes posições.

De Araujo T.B. et al. em 2013, avaliou 28 idosos com idades entre 60 e 84 anos, divididos em dois grupos $(\mathrm{GC}=$ sem intervenção e $\mathrm{GE}=$ Equoterapia $)$, avaliando a força muscular dos membros inferiores após 8 semanas de intervenção, com o teste de sentar e levantar da cadeira em 30 segundos (TSL), metodo indireito de avaliação de força muscular, segundo o estudo os idosos que foram submetidos ao programa de Equoterapia, tiveram um ganho na força muscular de membros inferiores estatitisticamente significativo $(p=0,032)$, quando comparado com o grupo controle que não recebeu intervenção. Assim, concorda com os resultados obtidos no presente estudo, que avaliou esse ganho com o dinamometro isocinetico (gold standart), tanto no GF como no GE.

Dentre das técnicas disponíveis para a abordagem de idosos com DA, a Fisioterapia Convencional (Cinesioterapia) é uma modalidade utilizada para manter ou melhorar a força 
muscular. Nas primeiras fases da doença um programa de exercícios com carga ajudam a prevenir problemas osteoarticulares e cardiovasculares.

Segundo a revisão sistematica de Liu, C. e Latham, N. K. em 2009, aonde foram incluídos 121 ensaios com 6700 idosos. Na maioria dos ensaios, o treinamento resistido progresivo (TRP) foi realizado duas a três vezes por semana e em uma alta intensidade. O TRP teve um efeito positivo grande na força muscular (73 ensaios, 3059 participantes, DMS 0,84, IC 95\% 0,67 a 1,00). Concordando assim com os resultados do presente estudo no que refere ao programa de Fisioterapia Convencional (Cinesioterapia), constatandose o ganho de força muscular nos membros inferiores de idosos com DA.

Num estudo que avaliou o efeito do treinamento resistido no comportamento cognitivo em idosas sedentarias, após 12 semanas de intervenção, mostrou que para o grupo experimental $(\mathrm{N}=29)$ que foi submetido a um programa de treinamento resistido que envolvia exercícios de membros inferiores combinados em $3 \times 10$ repetições com intervalo de 1 minuto entre repetições e dois minutos de repouso entre exercícios (três vezes / semana). Mostraram aumentos significativos na força muscular de membros inferiores (68\%) avaliados com o teste de sentar e levantar da cadeira em 30 segundos e na capacidade cognitiva (19\%). Aplicando exercicios para grupos musculares (adutor, abdutor, máquina extensora, máquina flexora e leg press) similares aos do presente estudo, no que se refere ao programa de Fisioterapia (DE CAMARGO, A., 2016).

Tanto o Programa de Equoterapia, quanto o Programa de Fisioterapia, tiveram uma adesão importante dos sujeitos, não se apresentando mortalidade amostral, uma das possiveis razões na adesão ao Programa de Fisioterapia, radica na constante motivação por parte dos fisioterapeutas, criando um ambiente estimulante e recursos terapeuticos amplos na confeção das sessões. Por outro lado, o Programa de Equoterapia, oferece por si só, um ambiente aberto, tranquilo, em contato com a natureza, gerando uma fidelização ao programa.

Destaca-se aqui que essas variáveis em espaços maiores de tempo poderiam gerar melhores análises quanto aos resultados e ganhos, logo um maior espaço de tempo seria necessario para se entender e analizar se as variávies mantem ou não a esperada autonomia funcional neste tipo de patologias neurodegenerativas.

Até o presente momento não foram encontrados estudos, que analisassem a força muscular com o isocinético em intervenções com Equoterapia em idosos com DA.

Sendo assim a ativação muscular vem sendo avaliada de outras formas. Em um estudo desenvolvido na Coreia com 30 idosos, que simularam em um protótipo mecânico de cavalo, a 
prática da Equoterapia, mesurando através de uma avaliação eletromiográfica à ativação da musculatura de tronco e quadril, comparado com um grupo controle que realizou uma terapia convencional. O grupo que praticou o programa simulado de Equoterapia teve um aumento na ativação da musculatura lombar baixa passando de ter $86.7 \%$ de ativação pré intervenção a 141,39\% de ativação pós intervenção, em relação ao glúteo médio, uns dos principais músculos estabilizadores de quadril adoptando-se um p-valor < 0,05, passou de ter $99.76 \%$ de ativação pré intervenção para $147.16 \%$ pós intervenção, embora, seja utilizado outro instrumento de avaliação constatouse da mesma forma que no presente estudo um aumento na ativação muscular avaliada o que em conjunto com os dados apresentados com a avaliação com o dinamometro isocinetico, tornase um complemento no que se refere à avaliações padrão ouro. (KIM, SEONG-GIL; LEE, JUNG-HO, 2015).

Como desfecho secundário que se considerou importante e que se relaciona com a pesquisa de McGibbon N. H. et al, 2009, é o tempo utilizado para a realização dos exercícios, precisando de apenas 10 minutos como mínimo, para obter ganho na força dos membros inferiores, pelo que demonstra ser uma modalidade de exercício de alta intensidade e de curta duração, considerando que o tempo utilizado no Programa de Equoterapia foi de 30 minutos. 


\section{Conclusões}

Com base nos dados apresentados, identificou-se uma relação entre a prática de Equoterapia e a Fisioterapia convencional no ganho de força muscular nos membros inferiores em sujeitos com doença de Alzheimer fase leve e moderada de ambos sexo.

Contudo, esta relação de ganho de força se manifestou nos índices de flexão de joelho para ambos grupos, levando-se em consideração que esses padrões de movimentos são altamente funcionais nas atividades da vida diária de idosos.

Ademais, foi possível identificar que a Equoterapia e a Fisioterapia convencional podem representar uma atividade física de intensidade moderada e alta, que estimulam grupos musculares específicos.

A importância da Fisioterapia é o fato de garantir uma opção de tratamento real para esta população especifica, garantindo um ambiente seguro e resultados que podem ser comprovados estatisticamente.

Por outro lado a Equoterapia é uma atividade física prazerosa desenvolvida em um ambiente estimulante para a prática de atividade física, com a interação do cavalo, criando mais um espaço e mais uma ferramenta terapêutica para este tipo de população, mostrando-se segura e viável de praticar.

\section{Limitações do estudo}

Considerando os resultados e que estes foram provenientes de uma amostra pequena e com baixo poder estatístico, recomendamos futuras investigações de maior duração e com maior número de indivíduos. 


\section{Referências Bibliográficas}

ABREU, I. D.; BARROS, H. L.; FORLENZA, O.V.; (2005). Demência de Alzheimer: correlação entre memória e autonomia. Revista de Psiquiatria Clínica. v. 32, n. 3, p. 131136, 2003.

AHN, N.; KIM, K. Effects of an elastic band resistance exercise program on lower extremity muscle strength and gait ability in patients with Alzheimer's disease. Journal of Physical Therapy Science, v. 27, n. 6, p. 1953-1955, 2015.

ALBERS, M. W. ET AL. At the interface of sensory and motor dysfunctions and Alzheimer's disease. Alzheimer's \& dementia : the journal of the Alzheimer's Association Volume 11, Issue 1, 70 - 28 Aug. 2016.

ALFARO-ACHA, A. et al. Handgrip strength and cognitive decline in older Mexican Americans. The Journals of Gerontology Series A: Biological Sciences and Medical Sciences, v. 61, n. 8, p. 859-865, 2006.

AMERICAN COLLEGE OF SPORTS MEDICEN Position Stand. Exercise and Physical Activity for Older Adults. Med Sci Sports Exerc,;41(7):1510-1530, 2009.

ANIANSSON, A.; GRIMBY, G.; HEDBERG, M. Compensatory muscle fiber hypertrophy in elderly men. Journal of Applied Physiology, v. 73, n. 3, p. 812-816, 1992.

ANTUNES F. ET AL. Different horse's paces during hippotherapy on spatio-temporal parameters of gait in children with bilateral spastic cerebral palsy: A feasibility study. Res Dev Disabil 2016 Aug 9;59:65-72, 2016

APRAHAMIAN, I.; MARTINELLI, J. E.; YASSUDA, M. S. Doença de Alzheimer: revisão da epidemiologia e diagnóstico. Rev Bras Clin Med, v. 7, n. 6, p. 27-35, 2009.

ARAÚJO T. B, ET AL. Efeito da Equoterapia no Equilíbrio Postural de Idosos. Rev Bras de Fisioterapia.;15(5):414-419,2011.

AVLUND K., ET AL. Maximal isometric muscle strength and functional ability in daily activities among 75-year-old men and women. Scandinavian journal of medicine \& science in sports, v. 4, n. 1, p. 32-40, 1994. 
BECKETT, M. W.; ARDERN, C. I.; ROTONDI, M. A. A meta-analysis of prospective studies on the role of physical activity and the prevention of Alzheimer's disease in older adults. BMC geriatrics, v. 15, n. 1, p. 1, 2015.

BENDA, W.; MCGIBBON, N. H.; GRANT, K. L. Improvements in muscle symmetry in children with cerebral palsy after equine-assisted therapy (hippotherapy). The Journal of Alternative \& Complementary Medicine, v. 9, n. 6, p. 817-825, 2003

BENTO, P. C. B. et al. Peak torque and rate of torque development in elderly with and without fall history. Clinical Biomechanics, v. 25, n. 5, p. 450-454, 2010.

BLOOM, D. E. 7 billion and counting. Science, v. 333, n. 6042, p. 562-569, 2011.

BLOCKER JR, W. P. Maintaining functional independence by mobilizing the aged. Geriatrics, v. 47, n. 1, p. 42, 48-50, 53 passim, 1992.

BOSSERS W.J et al. A nine-week-long aerobic and strength training program improves cognitive and motor function in patients with dementia: A randomized, controlled trial. Am J Geriatr Psychiatry. 2015; 23(11): 1106-1116. doi:10.1016/j.jagp.2014.12.191

BOTTARO, M.; RUSSO, A.; OLIVEIRA, R. J. The effects of rest interval on quadriceps torque during an isokinetic testing protocol in elderly. J Sports Sci Med, v. 4, n. 3, p. 28590, 2005.

BOTTARO, M. et al. Effect of high versus low-velocity resistance training on muscular fitness and functional performance in older men. European journal of applied physiology, v. 99, n. 3, p. 257-264, 2007.

BOYLE, P. A. et al. Association of muscle strength with the risk of Alzheimer disease and the rate of cognitive decline in community-dwelling older persons. Archives of neurology, v. 66, n. 11, p. 1339-1344, 2009.

BRIAN CLARK; TODD MANINI. Functional Consequences of Sarcopenia and Dynapenia in the Elderly. Curr Opin Clin Nutr Metab Care., p. 1-9, 2011.

BRONZUOLI, M. R. et al. Targeting neuroinflammation in Alzheimer's disease. Journal of Inflammation Research, v. 9, p. 199, 2016. 
BUCHMAN, A. S. et al. Change in body mass index and risk of incident Alzheimer disease. Neurology, v. 65, n. 6, p. 892-897, 2005.

BURNS, J. M. et al. Reduced lean mass in early Alzheimer disease and its association with brain atrophy. Archives of neurology, v. 67, n. 4, p. 428-433, 2010.

CARO, J. et al. To what degree does cognitive impairment in Alzheimer's disease predict dependence of patients on caregivers?. BMC neurology, v. 2, n. 1, p. 1, 2002.

CARVALHO, J.; SOARES, J. MC. Envelhecimento e força muscular: breve revisão. Revista Portuguesa de Ciências do Desporto, v. 4, n. 3, p. 79-93, 2004.

CARVALHO, J. A. M. D.; GARCIA, R. A. O envelhecimento da populaçäo brasileira: um enfoque demográfico. Cadernos de Saúde Pública, v. 19, n. 3, p. 725-733, 2003.

CIRILLO, L.C. Fundamentos doutrinários da Equoterapia no Brasil. In: Coletânea de Trabalhos do II Congresso Brasileiro de Equoterapia. São Paulo, 2002.

COPETTI, F. et al. Comportamento angular do andar de crianças com síndrome de Down após intervenção com equoterapia. Rev Bras Fisioter, v. 11, n. 6, p. 503-7, 2007.

CONDE-SALA, J. L. et al. [Cognitive decline in Alzheimer's disease. A follow three or more years of a sample of patients]. Revista de neurologia, v. 56, n. 12, p. 593-600, 2013.

CORREA, C. S. et al. 3 Different types of strength training in older women.International journal of sports medicine, v. 33, n. 12, p. 962-969, 2012.

DEBUSE, D; CHANDLER, C.; GIBB, C. An exploration of German and British physiotherapists' views on the effects of hippotherapy and their measurement. Physiotherapy theory and practice, v. 21, n. 4, p. 219-242, 2005.

DESCHENES, M. R. Effects of aging on muscle fibre type and size. Sports Medicine, v. 34, n. 12, p. 809-824, 2004.

DE AQUINO, R. et al. Abordagem fisioterapeutica no paciente portador de doença de Alzheimer: Revisão da Literatura. CORPVS/Rev. dos Cursos de Saúde da Faculdade Integrada do Ceará, Fortaleza, n25 p.39-44 Jan/Mar. 2013 
DE CAMARGO, A. The effects of strength training on cognitive performance in elderly women. Clinical Interventions in Aging, v. 11, p. 749-754, 2016.

DE CARVALHO, K.R. et al. O método Kabat no tratamento fisioterapêutico da doença de Alzheimer. Kairós Gerontologia. Revista da Faculdade de Ciências Humanas e Saúde. ISSN 2176-901X, v. 11, n. 2, 2009.

DE LIMA BORGES, L.; ALBUQUERQUE, C. R.; GARCIA, P. A.O impacto do declínio cognitivo, da capacidade funcional e da mobilidade de idosos com doença de Alzheimer na sobrecarga dos cuidadores. Fisioterapia e Pesquisa, v. 16, n. 3, p. 246-251, 2009.

DE MELO, M. A.; DRIUSSO, P. Proposta Fisioterapêutica para os cuidados de Portadores da Doença de Alzheimer. 2006. www.researchgate.net

DOUGHERTY, R. J. et al. Meeting physical activity recommendations may be protective against temporal lobe atrophy in older adults at risk for Alzheimer's disease. Alzheimer's \& Dementia: Diagnosis, Assessment \& Disease Monitoring, v. 4, p. 14-17, 2016.

DUNCAN, P. W. et al. How do physiological components of balance affect mobility in elderly men?. Archives of physical medicine and rehabilitation, v. 74, n. 12, p. 1343-1349, 1993.

ELY, J. C.; GRAVE, M. Estratégias de intervenção fisioterapêutica em indivíduo portador de doença de Alzheimer. Revista Brasileira de Ciências do Envelhecimento Humano, v. 5, n. 2, 2009.

ERICKSON, K.I.; LECKIE, R. L.; WEINSTEIN, A. M. Physical activity, fitness, and gray matter volume. Neurobiology of aging, v. 35, p. S20-S28, 2014.

ERICKSON, K. I.; WEINSTEIN, A. M.; LOPEZ, O. L. Physical activity, brain plasticity, and Alzheimer's disease. Archives of medical research, v. 43, n. 8, p. 615-621, 2012.

ESTRADA, M. et al. Functional impact of relative versus absolute sarcopenia in healthy older women. Journal of the American Geriatrics Society, v. 55, n. 11, p. 1712-1719, 2007.

FERREIRA, S. T. et al. Inflammation, defective insulin signaling, and neuronal

dysfunction in Alzheimer's disease. Alzheimer's \& Dementia, v. 10, n. 1, p. S76-S83, 2014. 
FORLENZA, O. V.; DINIZ, B. S.; GATTAZ, W. F. Diagnosis and biomarkers of predementia in Alzheimer's disease. BMC medicine, v. 8, n. 1, p. 1, 2010.

FROTA, N. A.F. et al. Critérios para o diagnóstico de doença de Alzheimer. Dement. neuropsychol, v. 5, n. supl 1, 2011.

FURMAN, J. M.; REDFERN, M. S. Effect of aging on the otolith-ocular reflex. Journal of Vestibular Research, v. 11, n. 2, p. 91-103, 2001.

GAGO, M. F. et al. Compensatory Postural Adjustments in an Oculus Virtual Reality Environment and the Risk of Falling in Alzheimer's Disease. Dementia and Geriatric Cognitive Disorders Extra, v. 6, n. 2, p. 252-267, 2016.

GARUFFI, M. et al. Atividade física para promoção da saúde de idosos com doença de Alzheimer e seus cuidadores. Revista Brasileira de Atividade Física \& Saúde, v. 16, n. 1, p. 80-83, 2012.

HARTMANN, A. et al. Reproducibility of an isokinetic strength-testing protocol of the knee and ankle in older adults. Gerontology, v. 55, n. 3, p. 259-268, 2008.

HENRY, W.; QUERFURTH, H. W.; LAFERLA, F. M. Mechanisms of disease Alzheimer's disease. New Engl J Med, v. 362, p. 329-44, 2010.

HURLEY, B. F.; HANSON, E. D.; SHEAFF, A. K. Strength training as a countermeasure to aging muscle and chronic disease. Sports Medicine, v. 41, n. 4, p. 289-306, 2011.

HYATT, R. H. et al. Association of muscle strength with functional status of elderly people. Age and ageing, v. 19, n. 5, p. 330-336, 1990.

GADELHA, A. B. et al. Effects of resistance training on sarcopenic obesity index in older women: A randomized controlled trial. Archives of gerontology and geriatrics, v. 65, p. 168173, 2016.

GRIMBY, G. et al. Training can improve muscle strength and endurance in 78-to 84-yrold men. Journal of Applied Physiology, v. 73, n. 6, p. 2517-2523, 1992.

HENWOOD, T. R.; TAAFFE, D. R. Detraining and retraining in older adults following long-term muscle power or muscle strength specific training. The Journals of Gerontology Series A: Biological Sciences and Medical Sciences, v. 63, n. 7, p. 751-758, 2008. 
HOMNICK, D. N. et al. Effect of therapeutic horseback riding on balance in communitydwelling older adults with balance deficits. The Journal of Alternative and Complementary Medicine, v. 19, n. 7, p. 622-626, 2013.

HUNTER, G. R.; MCCARTHY, J. P.; BAMMAN, M. M. Effects of resistance training on older adults. Sports medicine, v. 34, n. 5, p. 329-348, 2004.

INSTITUTO BRASILEIRO DE GEOGRAFIA E ESTATÍSTICA. Contas Nacionais. ContaSatélite de Saúde 2007- 2009. Rio de Janeiro, 2012.

Primeiros resultados definitivos do Censo 2010: população do Brasil é de 190.755.799 pessoas. Recuperado em 23 agosto, 2010.

JANURA, M. et al. Effect of repeated therapeutic horse riding sessions on the trunk movement of the rider. Neuroendocrinology Letters, v. 36, n. 5, p. 481-489, 2015.

JANURA, M. et al. An assessment of the pressure distribution exerted by a rider on the back of a horse during hippotherapy. Human movement science, v. 28, n. 3, p. 387-393, 2009.

JELLINGER, K. A.; ATTEMS, J. Neuropathological evaluation of mixed dementia. Journal of the neurological sciences, v. 257, n. 1, p. 80-87, 2007.

KATO, E. M. et al. Evaluation of the balance control in Alzheimer disease. Arq Neuropsiquiatr, v. 63, n. 2, p. S110, 2005.

KATO, E. M; RADANOVIC, M. Fisioterapia nas demências. Atheneu, 2007.

KIM, SEONG-GIL; LEE, JUNG-HO. The effects of horse riding simulation exercise on muscle activation and limits of stability in the elderly. Archives of gerontology and geriatrics, v. 60, n. 1, p. 62-65, 2015.

KIM, S. G.; LEE, Chae-Woo. The effects of hippotherapy on elderly persons' static balance and gait. Journal of physical therapy science, v. 26, n. 1, p. 25-27, 2014.

KOTTKE, F.J.; LEHMANN, J.F. Tratado de Medicina e Reabilitação de Krusen. 4 ed. São Pulo. Manole, 2002. 
KWON S., et al., Relationship between muscle strength and the time taken to complete a standardized walk-turn-walk test. The Journals of Gerontology Series A: Biological Sciences and Medical Sciences, v. 56, n. 9, p. B398-B404, 2001.

LAROCHE, D. P.; MILLETT, E. D.; KRALIAN, R. J. Low strength is related to diminished ground reaction forces and walking performance in older women. Gait \& posture, v. 33, n. 4, p. 668-672, 2011.

LENARDT, M. H. et al. Desempenho das atividades de vida diária em idosos com Alzheimer. Cogitare Enfermagem, v. 16, n. 1, 2011.

LETTS, Lori et al. Using occupations to improve quality of life, health and wellness, and client and caregiver satisfaction for people with Alzheimer's disease and related dementias. American Journal of Occupational Therapy, v. 65, n. 5, p. 497-504, 2011.

LINDROTH, J. L.; SULLIVAN, J. L.; SILKWOOD-SHERER, D. Does hippotherapy effect use of sensory information for balance in people with multiple sclerosis?. Physiotherapy theory and practice, v. 31, n. 8, p. 575-581, 2015.

LIU, C.; LATHAM, N. K. Progressive resistance strength training for improving physical function in older adults. The Cochrane Library, 2009.

LOPEZ, I.; HONRUBIA, V.; BALOH, R. W. Aging and the human vestibular nucleus. Journal of Vestibular Research, v. 7, n. 1, p. 77-85, 1997.

LOPEZ, O. L. et al. Predicting cognitive decline in Alzheimer's disease: an integrated analysis. Alzheimer's \& Dementia, v. 6, n. 6, p. 431-439, 2010.

MACALUSO, A.; DE VITO, G. Muscle strength, power and adaptations to resistance training in older people. European journal of applied physiology, v. 91, n. 4, p. 450-472, 2004.

MACDONALD, S. WS; DECARLO, C. A.; DIXON, R. A. Linking biological and cognitive aging: toward improving characterizations of developmental time. The Journals of Gerontology Series B: Psychological Sciences and Social Sciences, v. 66, n. suppl 1, p. i59i70, 2011. 
MACKEY, D. C.; ROBINOVITCH, S. N. Mechanisms underlying age-related differences in ability to recover balance with the ankle strategy.Gait \& posture, v. 23, n. 1, p. 59-68, 2006.

MAKI, B. E.; MCILROY, W. E. Postural control in the older adult.Clinics in geriatric medicine, v. 12, n. 4, p. 635-658, 1996.

MATOS, D. L. et al. Fatores sócio-demográficos associados ao uso de serviços odontológicos entre idosos brasileiros: um estudo baseado na Pesquisa Nacional por Amostra de Domicílios. Cad Saúde Pública, v. 20, n. 5, p. 1290-7, 2004.

MCGEE, M. C.; REESE, Nancy B. Immediate effects of a hippotherapy session on gait parameters in children with spastic cerebral palsy. Pediatric Physical Therapy, v. 21, n. 2, p. 212-218, 2009.

MCGIBBON, N. H. et al. Immediate and long-term effects of hippotherapy on symmetry of adductor muscle activity and functional ability in children with spastic cerebral palsy. Archives of physical medicine and rehabilitation, v. 90, n. 6, p. 966-974, 2009.

MEDEIROS, M. E.; RO, G. Tradução, adaptação e análises das propriedades psicométricas do activitiesofdaily living questionnaire (ADLQ) para avaliação funcional de pacientes com a doença de Alzheimer. Rev bras fisioter [periódico online], 2009.

MENDONÇA, G. M.S. de. Mal de alzheimer e a atuação fisioterapêutica. Monografia (Especialização) - Curso de Fisioterapia, Universidade Tiradentes, Tiradentes, 2011.

MINOSSO, J. S. M. et al. Validação, no Brasil, do Índice de Barthel em idosos atendidos em ambulatórios. Acta Paulista de Enfermagem, v. 23, n. 2, p. 218-223, 2010. MIRANDA, A. F. et al. Doença de Alzheimer: características e orientações em odontologia. RGO. Revista Gaúcha de Odontologia (Online), v. 58, n. 1, p. 103-107, 2010.

MÖLLER, U. O. et al. Prevalence and predictors of falls and dizziness in people younger and older than 80 years of age - a longitudinal cohort study. Archives of gerontology and geriatrics, v. 56, n. 1, p. 160-168, 2013.

MUÑOZ-LASA, S. et al. Effect of therapeutic horseback riding on balance and gait of people with multiple sclerosis. G Ital Med Lav Ergon, v. 33, n. 4, p. 462-467, 2011.

OKONKWO, O. C. et al. Physical activity attenuates age-related biomarker alterations in preclinical AD. Neurology, v. 83, n. 19, p. 1753-1760, 2014. 
PETERSON, M. D. et al. Resistance exercise for muscular strength in older adults: A meta-analysis. Ageing Research Reviews, v. 9, n. 3, p. 226-237, 2010.

PIERMARTIRI, T. CB; BEZERRA, N. C.; HOELLER, A. A. Efeito preventivo da fisioterapia na redução da incidência de quedas em pacientes com Doença de Alzheimer. Rev Neurocienc, v. 17, n. 4, p. 349-55, 2009.

PRINCE, M. et al. World Alzheimer Report 2015. The global impact of dementia. An analysis of prevalence, incidence, cost \& trends; Alzheimer's Disease International: London. 2015.

PROKOP, S.; MILLER, K. R.; HEPPNER, F. L. Microglia actions in Alzheimer's disease. Acta neuropathologica, v. 126, n. 4, p. 461-477, 2013.

QUEIROZ, Z. P. V.; LEMOS, N. de F. D.; RAMOS, L. R. Fatores potencialmente associados à negligência doméstica entre idosos atendidos em programa de assistência domiciliar. Cien Saude Colet, v. 15, n. 6, p. 2815-2824, 2010.

RAUCH, S. D. et al. Decreasing hair cell counts in aging humans.Annals of the New York Academy of Sciences, v. 942, n. 1, p. 220-227, 2001.

REBELLATO, J. R; MORELLI, J. G. da Silva. Fisioterapia geriátrica: a prática da assistência ao idoso. Manole, 2004.

REITZ, C.; BRAYNE, C.; MAYEUX, R. Epidemiology of Alzheimer disease. Nature Reviews Neurology, v. 7, n. 3, p. 137-152, 2011.

SANTOS, R. L. dos; JÚNIOR, J. S. V. Reliability of the Brazilian version of the Scale of Instrumental Activities of Daily Living.Revista Brasileira em Promocao da Saude, v. 21, n. 4, p. 290, 2008.

SCARMEAS, N. et al. Motor signs predict poor outcomes in Alzheimer disease. Neurology, v. 64, n. 10, p. 1696-1703, 2005.

SCHERDER, E. et al. Gait in ageing and associated dementias; its relationship with cognition. Neuroscience \& Biobehavioral Reviews, v. 31, n. 4, p. 485-497, 2007. 
SHUMWAY-COOK, A.; WOOLLACOTT, M. H. Controle motor: teoria e aplicações práticas. Manole, $3^{\mathrm{a}}$ Edição, 2010.

SINGH, M. A. F. Exercise comes of age rationale and recommendations for a geriatric exercise prescription. The Journals of Gerontology Series A: Biological Sciences and Medical Sciences, v. 57, n. 5, p. M262-M282, 2002

SUNWOO, Hyuk et al. Hippotherapy in adult patients with chronic brain disorders: a pilot study. Annals of rehabilitation medicine, v. 36, n. 6, p. 756-761, 2012.

SPERLING, R. A. et al. Toward defining the preclinical stages of Alzheimer's disease: Recommendations from the National Institute on Aging-Alzheimer's Association workgroups on diagnostic guidelines for Alzheimer's disease. Alzheimer's \& dementia, v. 7, n. 3, p. 280-292, 2011.

SPIRDUSO, W. W. Dimensões físicas do envelhecimento, ed. São Paulo: Manole, 2005.

STRAUSS I. et al. Hippotherapy: neurophysiological therapy on the horse.Thornhill, Ont: Ontario Therapeutic Riding Association, 1995.

SVOBODA, Z. et al. Does a rider influence a horse's movement in hippotherapy. Acta Univ Palacki Olomuc, v. 41, n. 4, p. 37-41, 2011.

SUNG, Y. et al. A hippotherapy simulator is effective to shift weight bearing toward the affected side during gait in patients with stroke. NeuroRehabilitation, v. 33, n. 3, p. 407$412,2013$.

TABBARAH, M; CRIMMINS, E. M.; SEEMAN, T. E. The relationship between cognitive and physical performance MacArthur Studies of Successful Aging. The Journals of Gerontology Series A: Biological Sciences and Medical Sciences, v. 57, n. 4, p. M228-M235, 2002.

TELL, G. S. et al. Relationship between balance and abnormalities in cerebral magnetic resonance imaging in older adults. Archives of Neurology, v. 55, n. 1, p. 73-79, 1998.

TOULOTTE C, FABRE C, DANGREMONT B, LENSEL G, THÉVENON A. Effects of physical training on the physical capacity of frail, demented patients with a history of falling: a randomised controlled trial. Age Ageing 2003;32(1):67-73. 
TROMBETTI, A. et al. Age-associated declines in muscle mass, strength, power, and physical performance: impact on fear of falling and quality of life. Osteoporosis international, v. 27, n. 2, p. 463-471, 2016.

VIGITEL, Brasil. Vigilância de fatores de risco e proteção para doenças crônicas por inquérito telefônico. SVS/Ministério da Saúde e NUPENS/Universidade de São Paulo, 2011.

VUKSANOVIC, M. et al. Cross-talk between muscle and bone in postmenopausal women with hypovitaminosis D. Climacteric, p. 1-6, 2016.

YUE, Guang $\mathrm{H}$. et al. Older adults exhibit a reduced ability to fully activate their biceps brachii muscle. The Journals of Gerontology Series A: Biological Sciences and Medical Sciences, v. 54, n. 5, p. M249-M253, 1999.

WANG, L. et al. Performance-based physical function and future dementia in older people. Archives of Internal Medicine, v. 166, n. 10, p. 1115-1120, 2006.

ZAIONS, J. C.; PAVAN, F. J.; WISNIEWSKI, M. S. W. A influência da Fisioterapia na preservação da memória e capacidade funcional de idoso portador de doença de Alzheimer: relato de caso. Rev Perspectiva, v. 36, p. 151-62, 2012.

ZIDAN, M. et al. Alterações motoras e funcionais em diferentes estágios da doença de Alzheimer. Revista de Psiquiatria Clínica, v. 39, n. 5, p. 161-5, 2012. 


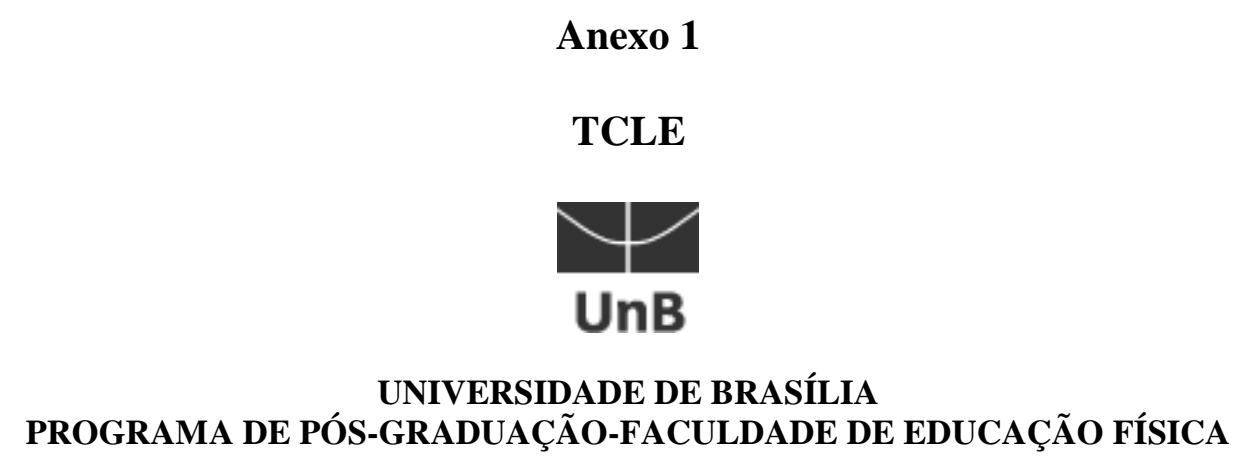

Termo de Consentimento Livre e Esclarecido - TCLE

Convidamos o(a) Senhor(a) a participar do projeto de pesquisa Efeito da Equoterapia na Aptidão Funcional, Memória e Funções Executivas de Idosos com Demência de Alzheimer Leve a moderada, sob a responsabilidade da pesquisadora Thais Borges de Araujo e mestrando Mauricio Ardiles Eguíluz. O projeto pretende estudar os efeitos da equoterapia no equilíbrio corporal, capacidade funcional e memória de idosos. Sabese que exercícios que trabalhem o equilíbrio e a flexibilidade, são fundamentais para a saúde da população idosa, considerada mais frágil devido às doenças crônicas prevalentes e o sedentarismo. Por isso, é importante que os pesquisadores e a comunidade descubram se a equoterapia é capazes de trazer benefícios para população, melhorando a qualidade de vida e diminuindo desta maneira o risco de queda e melhorando as funções executivas.

O objetivo dessa pesquisa é avaliar os efeitos de 20 sessões de equoterapia, sobre o equilíbrio, aptidão funcional memória e função executiva em idosos. Para isso, serão realizados questionários de identificação dos participantes, de avaliação da memória e da função executiva, além de testes de equilíbrio com uma plataforma de força. Durante os testes de equilíbrio o Senhor (a) irá subir e ficar em pé sobre uma plataforma horizontal, fixada no solo, 3 vezes, durante 30 segundos cada.

$\mathrm{O}$ (a) senhor(a) receberá todos os esclarecimentos necessários antes e no decorrer da pesquisa e lhe asseguramos que seu nome não aparecerá sendo mantido o mais rigoroso sigilo pela omissão total de quaisquer informações que permitam identificá-lo(a)

A sua participação se dará por meio de sessões de equoterapia que acontecerão gratuitamente no Instituto Cavalo Solidário, localizado no Núcleo Rural Alexandre Gusmão, Chácara 3/465 - DF. Os participantes serão transportados gratuitamente, por meio de um ônibus que sairá da Faculdade de Educação Física (FEF) da Universidade de Brasília com destino ao local aonde serão realizadas as sessões de equoterapia. As sessões terão duração aproximada de 30 minutos, serão realizadas durante 10 semanas, com uma freqüência de 2 sessões por semana e serão supervisionadas por uma equipe de professores de educação física e fisioterapia. É possível e normal que, nos primeiros dias, algumas pessoas sintam certo desconforto por causa da adaptação aos exercícios, como dores no corpo e cansaço naturais da pratica de atividade física.

O treinamento oferece riscos de queda, pois o cavalo é um animal irracional e pode ter reações que propiciem quedas. Contudo, visando minimizar os riscos de acidentes, todos os animais utilizados no estudo são treinados para esse tipo de terapia, além do treinamento do animal cada participante será guiado por um auxiliar guia treinado e terá mais 2 profissionais acompanhando o senhor de cada lado do animal. Essas medidas de segurança visam minimizar os riscos de acidentes. Como benefício, ao final da sequência das sessões de equoterapia espera-se melhoras no equilíbrio, capacidade funcional, memória e função executiva.

$\mathrm{O}$ (a) Senhor(a) pode se recusar a responder (ou participar de qualquer procedimento) qualquer questão que lhe traga constrangimento, podendo desistir de participar da pesquisa em qualquer momento sem nenhum prejuízo para o(a) senhor(a). Sua participação é voluntária, isto é, não há pagamento por sua colaboração.

Todas as despesas que você tiver relacionadas diretamente ao projeto de pesquisa (passagem para o local da pesquisa, alimentação no local da pesquisa ou exames para realização da pesquisa) serão cobertas pelo pesquisador responsável.

Caso haja danos diretos e indiretos decorrentes da pesquisa, você poderá ser indenizado, obedecendo-se as disposições legais vigentes no Brasil.

Os resultados da pesquisa serão divulgados na Universidade de Brasília podendo ser publicados posteriormente. Os dados e materiais utilizados na pesquisa ficarão sob a guarda do pesquisador por um período de no mínimo cinco anos, após isso serão destruídos ou mantidos na instituição.

Se o(a) Senhor(a) tiver qualquer dúvida em relação à pesquisa, por favor telefone para: Thais Borges de Araujo, na Universidade de Brasília no telefone (61)81656995 ou (61) 31072557 (no telefone fixo o horário de atendimento é de $8 \mathrm{hs}$ às $12 \mathrm{hs}$ e de $14 \mathrm{hs}$ às $17 \mathrm{hs}$, de segunda a sexta-feira). 
Este projeto foi Aprovado pelo Comitê de Ética em Pesquisa da Faculdade de Ciências da Saúde (CEP/FS) da Universidade de Brasília. O CEP é composto por profissionais de diferentes áreas cuja função é defender os interesses dos participantes da pesquisa em sua integridade e dignidade e contribuir no desenvolvimento da pesquisa dentro de padrões éticos. As dúvidas com relação à assinatura do TCLE ou os direitos do participante da pesquisa podem ser obtidos através do telefone: (61) 3107-1947 ou do e-mail cepfs@unb.br ou cepfsunb@gmail.com, horário de atendimento de das 10:00hs às 12:00hs e das 13:30hs às 15:30hs, de segunda a sexta-feira.

Este documento foi elaborado em duas vias, sendo que uma ficará com o pesquisador responsável e a outra com o Senhor(a) ou responsável legal.

Nome / assinatura/ (ou responsável Legal)

Pesquisador Responsável

Nome e assinatura

Brasília, de de 
Anexo 2

\section{Aprovação Comitê de Ética}

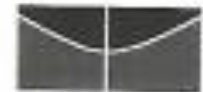 \\ Universidade de Brasilia \\ Faculdade de Ciências da Saúde \\ Comitê de Ética em Pesquisa - CEP/FS \\ PROCESSO DE ANÁLISE DE PROJETO DE PESQUISA}

Título do Projeto: "EFEITO DA EQUOTERAPIA NA APTIDĀO FUNCIONAL, MEMÓRIA E FUNÇŌES EXECUTIVAS DE IDOSOS COM DEMÉNCIA FASE

Pesquisadora Responsável: THAIS BORGES DE ARAUJO

Data de Entrada: 11/12/2014

CAAE: 36213314.9 .0000 .0030

Com base na Resoluçăo 466/12, do CNS/MS, que regulamenta a ética em pesquisa com seres humanos, o Comitê de Ética em Pesquisa com Seres Humanos da Faculdade de Ciências da Saúde da Universidade de Brasilia, após análise dos aspectos éticos e do contexto técnico-científico, resolveu APROVAR o projeto intitulado "EFEITO DA EQUOTERAPIA NA APTIDÃO FUNCIONAL, MEMÓRIA E FUNÇŌES EXECUTIVAS DE IDOSOS COM DEMÊNCIA FASE I". Parecer n 949.732, em 03 de fevereiro de 2015.

Notifica-se o(a) pesquisador(a) responsável da obrigatoriedade da apresentaçăo de um relatório semestral e relatório final sobre o desenvolvimento do projeto, no prazo de 1 (um) ano a contar da data de aprovaçāo.

Brasilia, 04 de março de 2015.

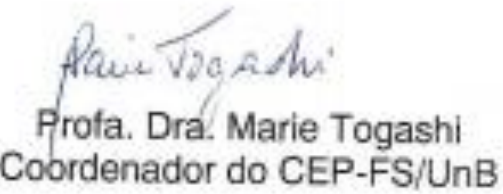


Anexo 3

Ficha de Avaliação da Força

\section{FICHA DE AVALIAÇÃO ISOCINETICA/ALZHEIMER}

Nome:

Peso:

altura:

idade:

Cuidador:

\begin{tabular}{|c|c|c|c|c|c|c|c|}
\hline Voluntario & ID\# & $\begin{array}{c}\text { Trein } \\
\text { o }\end{array}$ & $\begin{array}{c}\text { Profundida } \\
\text { de do } \\
\text { encosto }\end{array}$ & $\begin{array}{c}\text { Posição da } \\
\text { cadeira no } \\
\text { trilho }\end{array}$ & $\begin{array}{c}\text { Altura da } \\
\text { cadeira }\end{array}$ & $\begin{array}{c}\text { Posição do } \\
\text { eixo } \\
\text { dinamôme } \\
\text { tro }\end{array}$ & Braço \\
\hline PRÉ & & & & & & & \\
\hline PÓS & & & & & \\
\hline
\end{tabular}




\section{Anexo 4}

ESCALA DE BARTHEL

\begin{tabular}{|l|l|}
\hline \multicolumn{1}{|c|}{ ATIVIDADE } & PONTUAÇAO \\
\hline ALIMENTAÇAO & \\
$0=$ incapacitado \\
$5=$ precisa de ajuda para cortar, passar manteiga, etc, ou \\
dieta modificada \\
$10=$ independente & \\
\hline $\begin{array}{l}\text { BANHO } \\
0=\text { dependente } \\
5=\text { independente (ou no chuveiro) }\end{array}$ & \\
\hline $\begin{array}{l}\text { ATIVIDADES ROTINEIRAS } \\
0=\text { precisa de ajuda com a higiene pessoal } \\
5=\text { independente rosto/cabelo/dentes/barbear }\end{array}$ & \\
\hline $\begin{array}{l}\text { VESTIR-SE } \\
0=\text { dependente } \\
5=\text { precisa de ajuda mas consegue fazer uma parte sozinho } \\
10=\text { independente (incluindo botões, zipers, laços, etc.) }\end{array}$ & \\
\hline $\begin{array}{l}\text { INTESTINO } \\
0=\text { incontinente (necessidade de enemas) } \\
5=\text { acidente ocasional } \\
10=\text { continente }\end{array}$ & \\
\hline $\begin{array}{l}\text { SISTEMA URINARIO } \\
0=\text { incontinente, ou cateterizado e incapaz de manejo } \\
5=\text { acidente ocasional } \\
10=\text { continente }\end{array}$ & \\
\hline
\end{tabular}




\begin{tabular}{|c|c|}
\hline $\begin{array}{l}\text { USO DO TOILET } \\
0=\text { dependente } \\
5=\text { precisa de alguma ajuda parcial } \\
10=\text { independente ( pentear-se, limpar-se) }\end{array}$ & \\
\hline $\begin{array}{l}\text { TRANSFERENCIA (DA CAMA PARA A CADEIRA E } \\
\text { VICE VERSA) } \\
0=\text { incapacitado, sem equilibrio para ficar sentado } \\
5=\text { muita ajuda (uma ou duas pessoas, física), pode sentar } \\
10 \text { = pouca ajuda (verbal ou fisica) } \\
15=\text { independente }\end{array}$ & \\
\hline $\begin{array}{l}\text { MOBILIDADE (EM SUPERFICIES PLANAS) } \\
0=\text { imóvel ou }<50 \text { metros } \\
5=\text { cadeira de rodas independente, incluindo esquinas, }>50 \\
\text { metros } \\
10 \text { = caminha com a ajuda de uma pessoa (verbal ou física) }> \\
50 \text { metros } \\
15=\text { independente (mas pode precisar de alguma ajuda; } \\
\text { como exemplo, bengala) }>50 \text { metros }\end{array}$ & \\
\hline $\begin{array}{l}\text { ESCADAS } \\
0=\text { incapacitado } \\
5=\text { precisa de ajuda (verbal, física, ou ser carregado) } \\
10=\text { independente }\end{array}$ & \\
\hline
\end{tabular}

\begin{tabular}{|c|c|}
\hline Nivel de dependencia & Pontuação \\
\hline Total & 0 a 15 ptos \\
\hline Grave & 20 a 35 ptos \\
\hline Moderada & 40 a 55 ptos \\
\hline Leve & 60 a 95 ptos \\
\hline Independente & 100 ptos \\
\hline
\end{tabular}


Anexo 5

\section{MINIMENTAL}

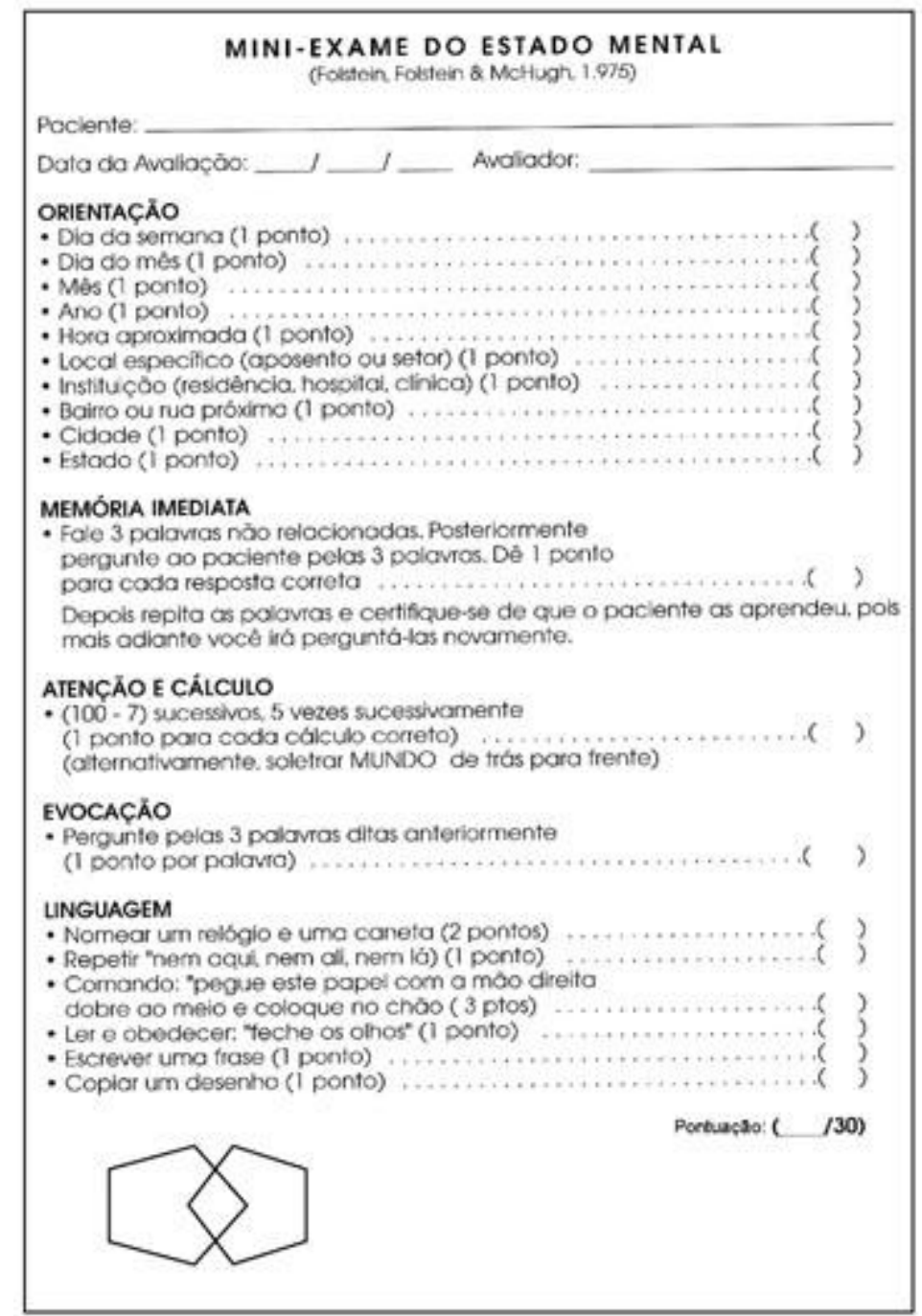

\section{Interpretação e escala de pontuação do \\ Mini Exame do Estado Mental (MEEM)}

Pontuaçăo Grau de escolaridade Diagnóstico

$\begin{array}{lll}<24 & \text { Alta ( } \geq 15 \text { anos) } & \text { Demência possivel } \\ <18 & \text { Média (8-11 anos) } & \text { Demência possivel } \\ <14 & \text { Baixa (4-7 anos) } & \text { Demência possivel } \\ \leq 8 & \text { Analfabeto } & \text { Demência possivel }\end{array}$

